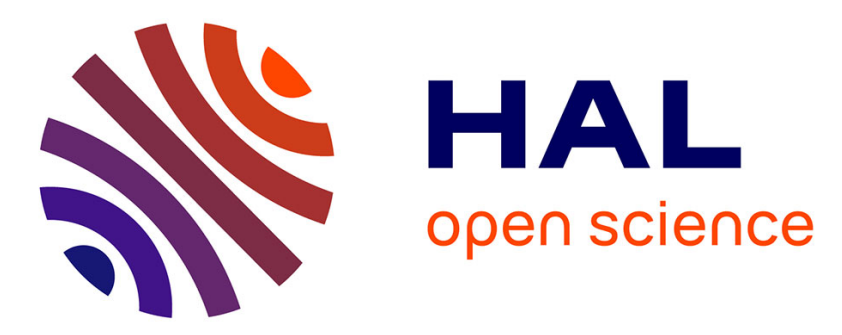

\title{
On distance-preserving elimination orderings in graphs: Complexity and algorithms
}

David Coudert, Guillaume Ducoffe, Nicolas Nisse, Mauricio Soto

\section{To cite this version:}

David Coudert, Guillaume Ducoffe, Nicolas Nisse, Mauricio Soto. On distance-preserving elimination orderings in graphs: Complexity and algorithms. Discrete Applied Mathematics, 2018, 243, pp.140153. 10.1016/j.dam.2018.02.007 . hal-01741277

\section{HAL Id: hal-01741277 \\ https://hal.inria.fr/hal-01741277}

Submitted on 22 Mar 2018

HAL is a multi-disciplinary open access archive for the deposit and dissemination of scientific research documents, whether they are published or not. The documents may come from teaching and research institutions in France or abroad, or from public or private research centers.
L'archive ouverte pluridisciplinaire HAL, est destinée au dépôt et à la diffusion de documents scientifiques de niveau recherche, publiés ou non, émanant des établissements d'enseignement et de recherche français ou étrangers, des laboratoires publics ou privés. 


\title{
On distance-preserving elimination orderings in graphs: complexity and algorithms*
}

\author{
David Coudert ${ }^{1}$, Guillaume Ducoffe ${ }^{2,3}$, Nicolas Nisse $^{1}$, and Mauricio Soto ${ }^{4}$ \\ ${ }^{1}$ Université Côte d'Azur, Inria, CNRS, I3S, France \\ ${ }^{2}$ ICI - National Institute for Research and Development in Informatics, Romania \\ ${ }^{3}$ The Research Institute of the University of Bucharest ICUB \\ ${ }^{4}$ Departamento de Ingeniera Matemàtica, Universidad de Chile. Facultad de Ciencias Fsicas y Matemàticas
}

\begin{abstract}
For every connected graph $G$, a subgraph $H$ of $G$ is isometric if the distance between any two vertices in $H$ is the same in $H$ as in $G$. A distance-preserving elimination ordering of $G$ is a total ordering of its vertex-set $V(G)$, denoted $\left(v_{1}, v_{2}, \ldots, v_{n}\right)$, such that any subgraph $G_{i}=G \backslash\left(v_{1}, v_{2}, \ldots, v_{i}\right)$ with $1 \leq i<n$ is isometric. This kind of ordering has been introduced by Chepoi in his study on weakly modular graphs [11]. We prove that it is NP-complete to decide whether such ordering exists for a given graph - even if it has diameter at most 2 . Then, we prove on the positive side that the problem of computing a distance-preserving ordering when there exists one is fixed-parameter-tractable in the treewidth. Lastly, we describe a heuristic in order to compute a distance-preserving ordering when there exists one that we compare to an exact exponential time algorithm and to an ILP formulation for the problem.
\end{abstract}

Keyword: distance-preserving elimination ordering; metric graph theory; NP-complete; exact exponential algorithm; integer linear programming; bounded treewidth.

\section{Introduction}

Elimination orderings of a graph are total orderings of its vertex-set. Many interesting graph problems can be specified in terms of the existence of an elimination ordering with some given properties. These range from some practical problems in molecular biology and chemistry [8] to the analysis of graph search algorithms [14], the characterization of some graph classes [10, 29], and the study of network clustering methods in social networks [26]. On the computational point of view, vertex ordering characterizations of a given graph class often lead to efficient (polynomialtime) recognition algorithms for the graphs in this class $[2,6,15,21,28]$. In this work we will consider one specific kind of elimination ordering that is called distance-preserving elimination ordering [11]. Precisely, let us remind that a subgraph $H$ of a graph $G$ is isometric if the distance between any two vertices in $H$ is the same in $H$ as in $G$. An elimination ordering $\left(v_{1}, v_{2}, \ldots, v_{n}\right)$ of $G$ is distance-preserving if it satisfies that each suffix $\left(v_{i}, v_{i+1}, \ldots, v_{n}\right)$ with $i<n$ induces an isometric subgraph of $G$.

${ }^{*}$ This work has been supported by ANR project Stint under reference ANR-13-BS02-0007, ANR program "Investments for the Future" under reference ANR-11-LABX-0031-01, and the Inria associated team AlDyNet. 
Distance-preserving elimination orderings encompass several other elimination orderings studied in the literature $[6,7,19,24,25,28]$, all of which can be computed in polynomial time when they exist. In particular, known refinements of distance-preserving elimination orderings comprise the perfect elimination orderings [28], maximum neighbourhood orderings [6], h-extremal orderings [7], semisimplicial elimination orderings [24], dismantlable orderings [25] and more generally domination elimination orderings [19]. The latter orderings characterize chordal graphs, dually chordal graphs, homogeneously orderable graphs, cop-win graphs and a subclass of tandem-win graphs [12] respectively, and as above stated they all can be computed in polynomial-time when they exist. However the complexity of deciding whether a distance-preserving elimination ordering exists in a given graph has been left open until this paper. We aim at completing the picture and characterizing the complexity of this problem.

Related work In [17] it has been proved that every graph with a distance-preserving elimination ordering has a minimum-size cycle basis with only triangles and quadrangles, that can be easily computed if a distance-preserving elimination ordering is part of the input. This property has been useful in the study of some tree-likeness invariants of graphs (e.g., in comparing treewidth with treelength). However, the complexity of recognizing graphs with a distance-preserving elimination ordering has been left open in [17]. Prior works [9, 11] have focused on the existence of distancepreserving elimination orderings in some well-structured graph classes, i.e., the weakly modular graphs. In particular, it has been proved recently in [9] that every breadth-first search ordering of a weakly modular graph is distance-preserving, that allows to compute one such ordering in linear time for a given graph in this class.

On the positive side, above stated refinements of distance-preserving elimination orderings [6, $7,19,24,25,28]$ can all be computed with greedy algorithms when they exist. Indeed, for all these orderings it can be tested in polynomial-time whether a given vertex can be eliminated first. As an example, any dominated vertex can be the starting vertex of some domination elimination ordering (total ordering of the vertex-set where for every suffix, the closed neighbourhood of the first vertex is dominated in the subgraph induced by the suffix). The latter implies that any partial domination elimination ordering can be extended unless the graph does not admit such a total order. A first hint that computing a distance-preserving elimination ordering can be more difficult is that it is not that simple to choose a starting vertex. For instance, consider the wheel $W_{5}$ obtained from a cycle $C_{5}$ of length five by adding a universal vertex. Every elimination ordering of $W_{5}$ where the universal vertex is the last vertex eliminated is distance-preserving. However, if the universal vertex is eliminated first then the cycle $C_{5}$ is an isometric subgraph of $W_{5}$ that does not admit a distance-preserving elimination ordering. The above problem occuring with $C_{5}$ also occurs with hypercubes, that can be proved using tools from discrete geometry ${ }^{1}$.

Our contributions We prove on the negative side that it is NP-complete to decide whether a given graph admits a distance-preserving elimination ordering (Section 3). The latter result may look surprising since as above stated, a broad range of distance-preserving orderings with additional properties can be computed in polynomial time when they exist. Then we show that the

\footnotetext{
${ }^{1}$ More precisely, for the special case of an $n$-dimensional hypercube, the distance-preserving orderings are equivalent to the so-called "shellable orderings" as defined in [32]. In particular, if every partial distance-preserving ordering of the $n$-dimensional hypercube could be extended, then it would imply that its dual, the $n$-dimensional octahedron, is extendably shellable, that is known to be false for $n \geq 12$ [22].
} 
problem remains NP-complete even for general graphs with diameter at most two (Section 3.3). Note that in a sense our result is optimal w.r.t. the diameter because complete graphs trivially admit a distance-preserving ordering. Our reduction will show how to encode a 3-SAT formula in a graph whose distance-preserving orderings are in many-to-many correspondance with the satisfying assignments for the formula. This line of work resembles to the one in [31] in order to show that it is NP-complete to recognize collapsible complexes. Our work differs from theirs in that we study orderings with very distinct properties and the "simpler" structure of graphs — w.r.t. complexesfurther constrains our gadgets to mimic variables and clauses of the formula.

On a more positive side, we prove in Section 4 that the problem of computing a distancepreserving ordering when there exists one is fixed-parameter-tractable in the treewidth.

Next, we show that a meta-theorem on vertex-orderings [3] can be applied to our problem, that results in an algorithm with $\mathcal{O}^{*}\left(2^{n}\right)$-time and space complexity, as well as in an algorithm with $\mathcal{O}^{*}\left(4^{n}\right)$-time and polynomial space complexity. We also propose an Integer Linear Programming formulation which may lead to a better running time in practice. These exact algorithms are described in Section 5 as well as simple heuristic algorithms.

Notations Graphs in this study are finite, simple (hence without loop nor multiple edges) and unweighted. We refer to [5, 20] for standard reference books on graphs (see also [1] for a survey about metric graph theory). Let $\left(v_{1}, v_{2}, \ldots, v_{n}\right)$ be an elimination ordering of a graph $G$, we say that vertex $v_{i}, 1 \leq i \leq n$, is the $i^{\text {th }}$ vertex to be eliminated, and that vertex $v_{i}$ is eliminated before vertex $v_{j}$, denoted $v_{i} \prec v_{j}$, if $i<j$.

\section{Local characterization}

In what follows, we will avoid considering all the distances in the graph at each time a vertex is eliminated. That is, we replace the "global" condition that $G \backslash v$ is isometric by a "local" one implying only the neighbours of $v$. The following characterization will explain how to do so.

Lemma 1. Let $G=(V, E)$ and $v \in V$, the subgraph $G \backslash v$ is isometric if and only if every two non-adjacent neighbours of vertex $v$ have at least two common neighbours in $G$ (including $v$ ).

Proof. If $G \backslash v$ is isometric, then let $x, y \in N_{G}(v)$ be non-adjacent. Since $\mathrm{d}_{G \backslash v}(x, y)=\mathrm{d}_{G}(x, y)=2$, $x$ and $y$ have another common neighbour than vertex $v$. Conversely, suppose that every two nonadjacent neighbours of vertex $v$ have at least two common neighbours in $G$. In particular, every of them have at least one common neighbour in $G \backslash v$. Then, for every two non-adjacent $x, y \in N_{G}(v)$ the subpath $(x, v, y)$ can be substituted in any shortest-path of $G$ with the subpath $(x, u, y)$ of $G \backslash v$, where $u$ denotes a common neighbour of $x, y$. This proves that $G \backslash v$ is an isometric subgraph.

By using Lemma 1, one obtains the following characterization of distance-preserving elimination orderings. It can be seen as a reformulation of the characterization given in [11, Lemma 3.2] in terms of pseudopeakless functions.

Corollary 2. An elimination ordering $\prec$ of $G=(V, E)$ is distance-preserving if and only if for every $u, v \in V$ at distance $\mathrm{d}_{G}(u, v)=2$, there is $w \in N_{G}(u) \cap N_{G}(v)$ such that $u \prec w$ or $v \prec w$.

Proof. Let $\left(v_{1}, v_{2}, \ldots, v_{n}\right)$ be the elimination ordering we consider. For every $0 \leq i<n$, define $G_{i}=G \backslash\left\{v_{1}, \ldots, v_{i-1}\right\}$ (in particular $G_{0}=G$ ). On the one direction, suppose that $\prec$ is distancepreserving. Let $v_{i}, v_{j} \in V$ satisfy $\mathrm{d}_{G}\left(v_{i}, v_{j}\right)=2$ with $i<j$. Since $\prec$ is distance-preserving, 
$G_{i}$ is an isometric subgraph of $G$. Hence, since $v_{i}, v_{j} \in V\left(G_{i}\right)$ and $\mathrm{d}_{G}\left(v_{i}, v_{j}\right)=2$, there exists $w \in N_{G}\left(v_{i}\right) \cap N_{G}\left(v_{j}\right)$ such that $w \in V\left(G_{i}\right)$, i.e., $v_{i} \prec w$. On the other direction, suppose that $\prec$ is not distance-preserving. Let $i \geq 0$ be the least index such that $G_{i}$ is an isometric subgraph of $G$ but $G_{i+1}=G_{i} \backslash v_{i}$ is not. By Lemma 1, there exist $x, y \in N_{G_{i}}\left(v_{i}\right)$ nonadjacent such that $N_{G_{i}}(x) \cap N_{G_{i}}(y) \cap V\left(G_{i+1}\right)=\emptyset$. In particular, there does not exist any $w \in N_{G}(x) \cap N_{G}(y)$ such that $x \prec w$ or $y \prec w$ (else, $\left.w \in V\left(G_{i+1}\right)\right)$.

Finally, it may be easier sometimes to group vertices into subsets whose vertices can be eliminated in an arbitrary way. On such occasions, we will base on the following consequence of Lemma 1.

Corollary 3. Let $G$ be a graph, $S \subset V(G)$ satisfy that for every $v \in S$, every two non-adjacent neighbours of vertex $v$ have a common neighbour in $G \backslash S$. Then, for any $S^{\prime} \subseteq S$, the subgraph $G \backslash S^{\prime}$ is isometric.

Proof. By contradiction, let $S^{\prime} \subseteq S$ falsify the corollary with $S^{\prime}$ being of minimum size w.r.t. this property. Let $v \in S^{\prime}, S^{\prime \prime}:=S^{\prime} \backslash v$. The subgraph $G \backslash S^{\prime \prime}$ is isometric by the minimality of $S^{\prime}$. Furthermore, by the hypothesis every two non-adjacent neighbours of $v$ have a common neighbour in $G \backslash S$, hence in $G \backslash S^{\prime}$ so, $G \backslash S^{\prime}$ is isometric by Lemma 1. This contradicts the fact that $S^{\prime}$ falsifies the corollary.

\section{Hardness results}

The purpose of this section is to prove the following result.

Theorem 4. Deciding whether a given graph $G$ admits a distance-preserving elimination ordering is NP-complete, already if $G$ has diameter at most two.

Note that since the all-pairs-shortest-paths in a graph can be computed in polynomial-time then it easily follows that the problem is in NP and so, we will only prove the NP-hardness. We will first prove that deciding whether a given graph $G$ admits a distance-preserving elimination ordering is NP-hard, already if $G$ has diameter at most five. This first part of the proof is involved and it is based on a technical reduction from 3-SAT, the standard NP-complete problem [13]. Then, we will show how to lower the diameter to two (Section 3.3).

\subsection{Main reduction}

Given a formula $\Phi$ with $n$ variables and $m$ clauses of exactly three literals each, the 3 -SAT problem aims at deciding whether there exists a boolean assignment of the variables which makes the formula true. In case it does, then the formula $\Phi$ is said satisfiable. We will construct a graph $G_{\Phi}$ from an arbitrary formula $\Phi$ so that there is a distance-preserving elimination ordering of $G_{\Phi}$ if and only if $\Phi$ is satisfiable. This will prove the NP-hardness of our problem. To achieve the result, assume w.l.o.g. that no litteral and its negation can be contained in the same clause of $\Phi$ (else, any such clause could be removed from $\Phi$ ), and every variable appears both positively and negatively in the clauses of $\Phi$ (else, any clause containing either this variable or its negation could also be removed from $\Phi)$. Let us denote by $x_{1}, x_{2}, \ldots, x_{n}$ the $n$ variables, and by $C_{1}, C_{2}, \ldots, C_{m}$ the $m$ clauses of $\Phi$. The graph $G_{\Phi}$ is defined as follows. 
Variable gadget For every variable $x_{i}, 1 \leq i \leq n$, let us add in $G_{\Phi}$ an induced quadrangle $\left(x_{i}, y_{i}, \bar{x}_{i}, \bar{y}_{i}\right)$ (i.e., a cycle with four vertices). For every $1 \leq j \leq m$, if $x_{i}$ is in the $j^{\text {th }}$ clause of the formula then four more vertices $a_{i j}, b_{i j}, c_{i j}, d_{i j}$ are added and made adjacent to vertex $x_{i}$. Similarly if $\bar{x}_{i}$ is in the $j^{\text {th }}$ clause of the formula then four more vertices $a_{i j}, b_{i j}, c_{i j}, d_{i j}$ are added and made adjacent to vertex $\bar{x}_{i}$ (this is clearly defined because no clause contains both literals $x_{i}, \overline{x_{i}}$ by the hypothesis). We refer to Figure 1 for an illustration.
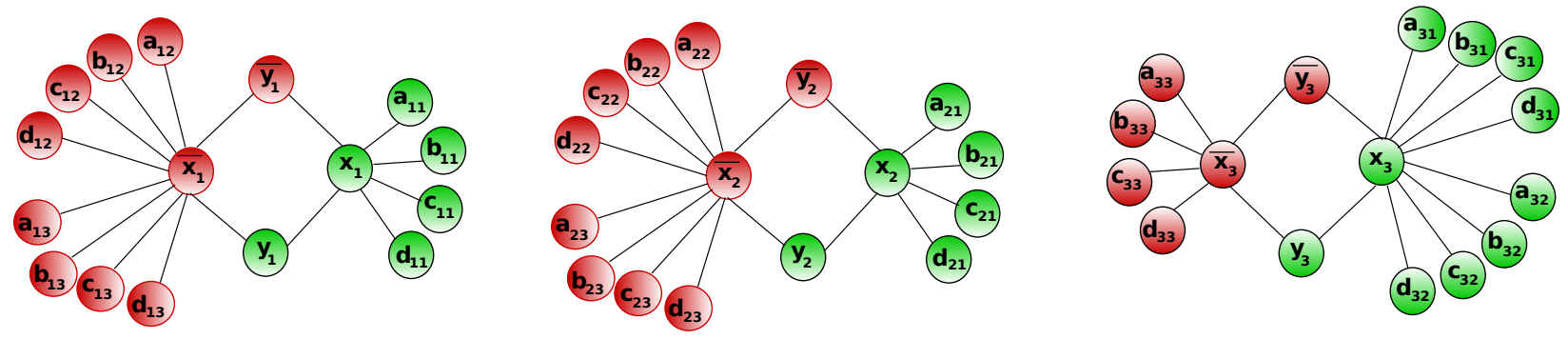

Figure 1: The three variable gadgets for the formula $\Phi=\left(x_{1} \vee x_{2} \vee x_{3}\right) \wedge\left(\overline{x_{1}} \vee \overline{x_{2}} \vee \overline{x_{3}}\right) \wedge\left(\overline{x_{1}} \vee \overline{x_{2}} \vee x_{3}\right)$

To better understand the role played by the quadrangle $\left(x_{i}, y_{i}, \bar{x}_{i}, \bar{y}_{i}\right)$ in our reduction, we make the following observation that captures well the difficulty of the problem. Indeed, every vertex in a quadrangle can be chosen as the starting vertex of a distance-preserving ordering. However, the vertex diametrically opposed cannot be chosen as the second vertex to be eliminated. We will make use of a similar trick in our reduction so as to mimic a truth table with variable gadgets, ensuring that the second vertex to be eliminated in $x_{i}, \overline{x_{i}}$ must be eliminated after one of each pair $x_{i^{\prime}}, \overline{x_{i^{\prime}}}$ has already been eliminated for any $1 \leq i^{\prime} \leq n$.

Clause tree Second, a rooted tree of depth two with $8 m+1$ vertices is added in $G_{\Phi}$. More precisely, the tree is rooted at some newly added vertex $r_{\Phi}$ that has $2 m$ children denoted by $s_{1}, t_{1}, s_{2}, t_{2}, \ldots, s_{m}, t_{m}$. Informally, for every $1 \leq j \leq m$ both nodes $s_{j}, t_{j}$ represent the $j^{\text {th }}$ clause of $\Phi$. Moreover let $C_{j}=l_{p} \vee l_{q} \vee l_{r}$ with $p<q<r$ and $l_{i} \in\left\{x_{i}, \bar{x}_{i}\right\}$ for every $i \in\{p, q, r\}$. Then, the internal node $s_{j}$ has three children denoted by $u_{j}(p, q), u_{j}(q, r)$ and $u_{j}(r, p)$, similarly the internal node $t_{j}$ has three children denoted by $v_{j}(p, q), v_{j}(q, r)$ and $v_{j}(r, p)$. Finally, let us describe how the clause tree is linked to the variable gadgets. Precisely, any leaf node $u_{j}(p, q)$ is made adjacent to the pair of vertices $a_{p j}, b_{q j}$, and in the same way any leaf node $v_{j}(p, q)$ is made adjacent to the pair of vertices $c_{p j}, d_{q j}$. We refer to Figure 2 for an illustration.

Our reduction will ensure that $r_{\Phi}$ is the unique common neighbour of $s_{j}, t_{j}$ in $G_{\Phi}$. Consequently, by Corollary 2 in any distance-preserving ordering of $G_{\Phi}$ one of $s_{j}, t_{j}$ will need to precede vertex $r_{\Phi}$. We will show that this implies that the $j^{\text {th }}$ clause of $\Phi$ is satisfied.

Literal clique The final and most technical part of our reduction is to construct a clique of $G_{\Phi}$ with $8 n$ vertices so as to ensure that a distance-preserving ordering exists if $\Phi$ is satisfiable. For every $1 \leq i \leq n$, the clique contains four vertices denoted by $e_{i}, f_{i}, g_{i}, h_{i}$ (related to variable $x_{i}$ ). In the same way there are four vertices denoted by $\bar{e}_{i}, \bar{f}_{i}, \bar{g}_{i}, \bar{h}_{i}$ (related to the negated variable $\bar{x}_{i}$ ).

This clique is connected to variable gadgets as follows. Vertex $y_{i}$ (in the $i^{\text {th }}$ variable gadget) is made adjacent to each of the four vertices $e_{i}, f_{i}, g_{i}, h_{i}$, and in the same way vertex $\bar{y}_{i}$ is made adjacent to each of the four vertices $\bar{e}_{i}, \bar{f}_{i}, \bar{g}_{i}, \bar{h}_{i}$. For every $1 \leq j \leq m$ such that one of $x_{i}, \bar{x}_{i}$ is a 


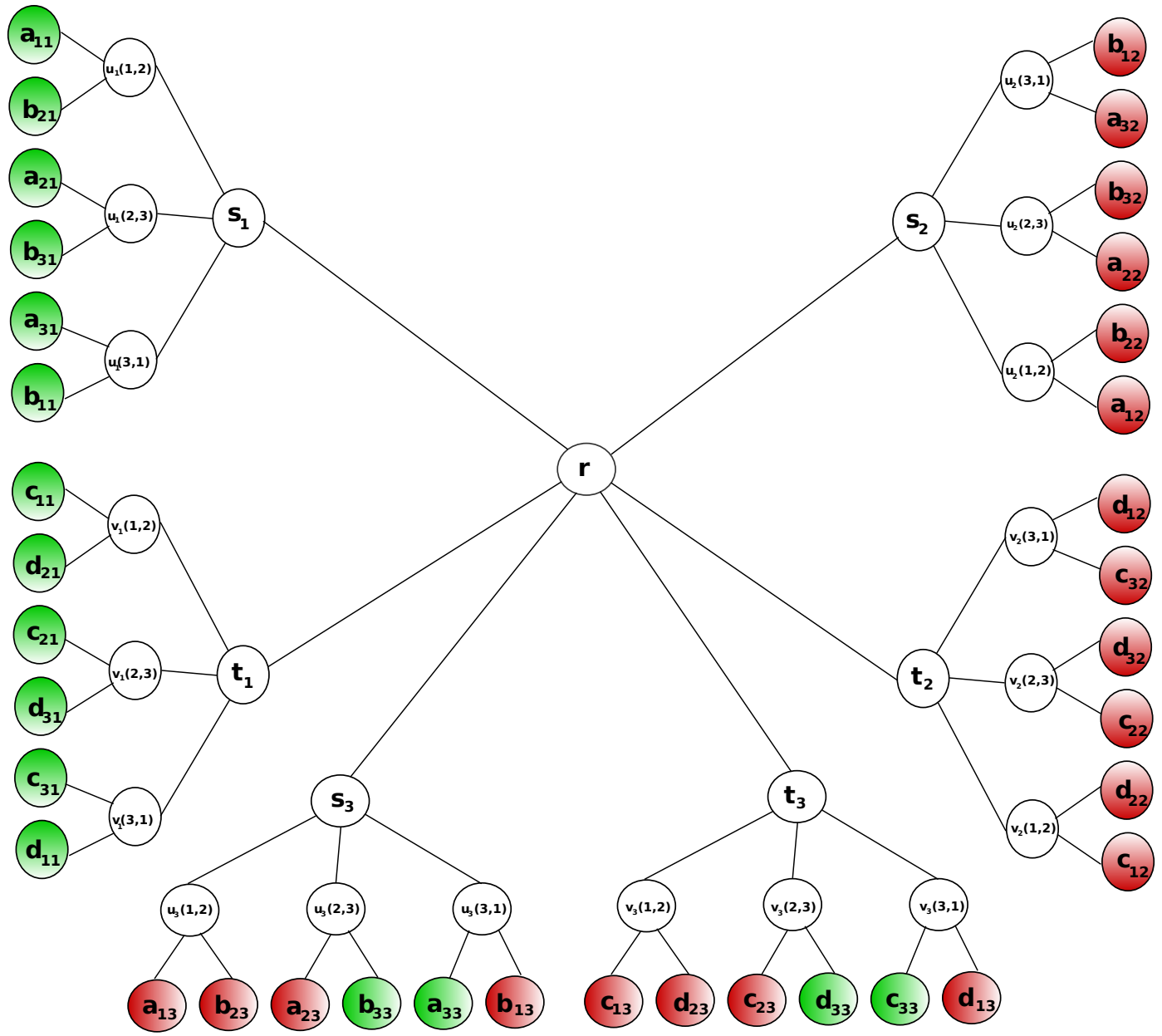

Figure 2: The clause tree for the formula $\Phi=\left(x_{1} \vee x_{2} \vee x_{3}\right) \wedge\left(\overline{x_{1}} \vee \overline{x_{2}} \vee \overline{x_{3}}\right) \wedge\left(\overline{x_{1}} \vee \overline{x_{2}} \vee x_{3}\right)$.

literal of $C_{j}$, the four vertices $a_{i j}, b_{i j}, c_{i j}$ and $d_{i j}$ are made adjacent to each of the four vertices $e_{i}, f_{i}$ and $\bar{e}_{i}, \bar{f}_{i}$.

Then, the clique is connected to the clause tree as follows. For any $1 \leq j \leq m$, let $C_{j}=l_{p} \vee l_{q} \vee l_{r}$ with $p<q<r$ and $l_{i} \in\left\{x_{i}, \bar{x}_{i}\right\}$ for every $i \in\{p, q, r\}$, then:

- the three vertices $u_{j}(p, q), u_{j}(q, r), u_{j}(r, p)$ are respectively made adjacent to the 4 -tuples of vertices: $\left(e_{p}, g_{p}\right.$ and $\left.\overline{e_{q}}, \overline{g_{q}}\right) ;\left(e_{q}, g_{q}\right.$ and $\left.\overline{e_{r}}, \overline{g_{r}}\right) ;\left(e_{r}, g_{r}\right.$ and $\left.\overline{e_{p}}, \overline{g_{p}}\right)$;

- similarly, the three vertices $v_{j}(p, q), v_{j}(q, r), v_{j}(r, p)$ are respectively made adjacent to the 4-tuples of vertices: $\left(f_{p}, h_{p}\right.$ and $\left.\bar{f}_{q}, \bar{h}_{q}\right) ;\left(f_{q}, h_{q}\right.$ and $\left.\bar{f}_{r}, \bar{h}_{r}\right) ;\left(f_{r}, h_{r}\right.$ and $\left.\bar{f}_{p}, \bar{h}_{p}\right)$;

- last, vertex $s_{j}$ is made adjacent to the twelve vertices $e_{i}, g_{i}$ and $\bar{e}_{i}, \bar{g}_{i}$ with $i \in\{p, q, r\}$; similarly, vertex $t_{j}$ is made adjacent to the twelve vertices $f_{i}, h_{i}$ and $\bar{f}_{i}, \bar{h}_{i}$ with $i \in\{p, q, r\}$.

Let $\mathcal{E}=\bigcup_{1 \leq i \leq n}\left\{e_{i}, \bar{e}_{i}\right\}, \quad \mathcal{F}=\bigcup_{1 \leq i \leq n}\left\{f_{i}, \bar{f}_{i}\right\}, \mathcal{G}=\bigcup_{1 \leq i \leq n}\left\{g_{i}, \bar{g}_{i}\right\}$ and $\mathcal{H}=\bigcup_{1 \leq i \leq n}\left\{h_{i}, \bar{h}_{i}\right\}$ partition the clique. The root vertex $r_{\Phi}$ of the clause tree is made adjacent to every vertex in $\mathcal{G} \cup \mathcal{H}$. We refer to Figure 3 for a partial illustration. 


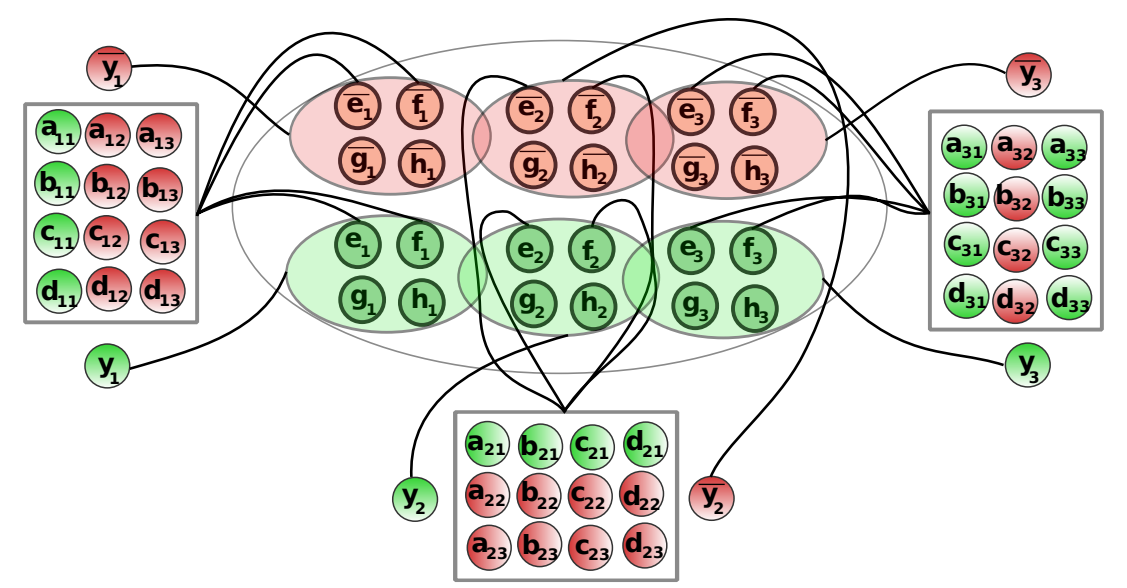

(a) Adjacency relations between vertices from the variable gadgets and those from the literal clique.

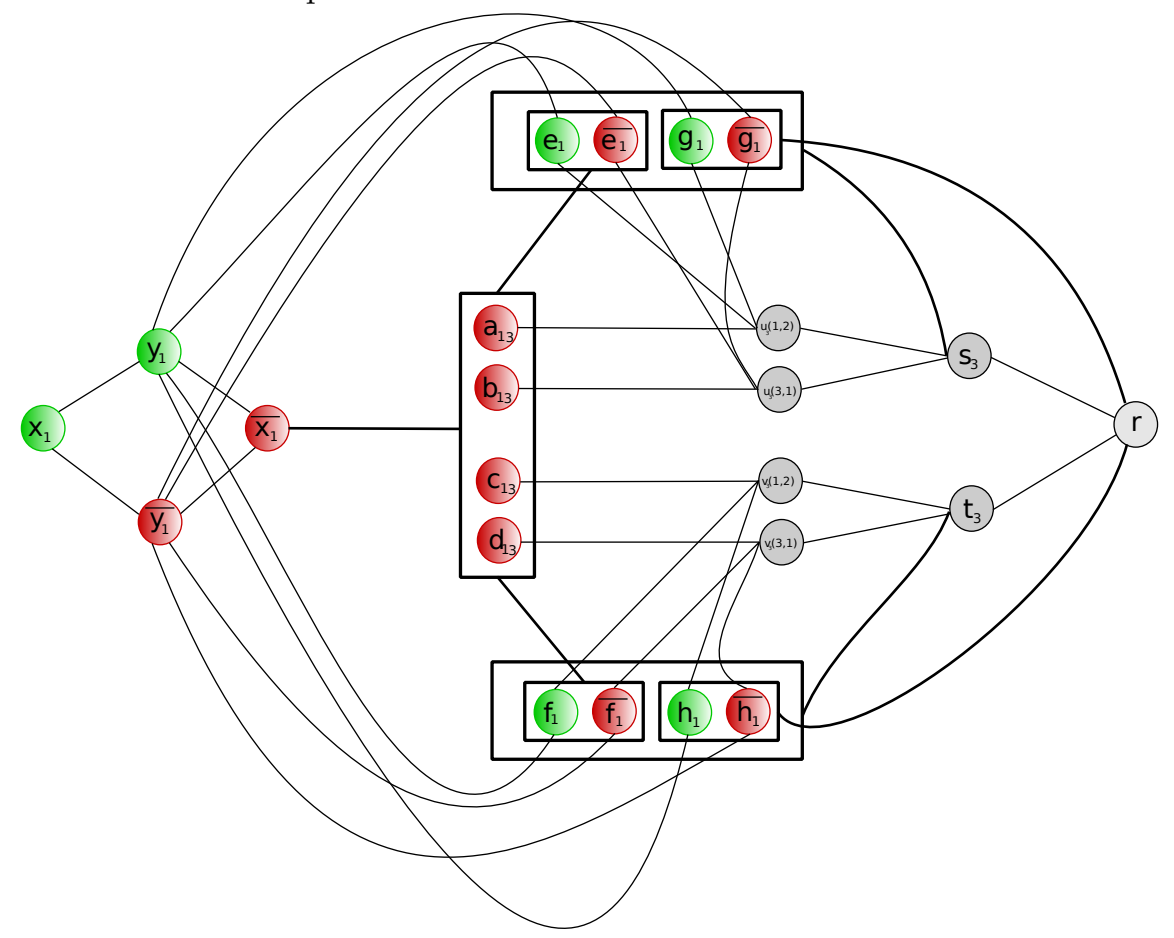

(b) Adjacency relations w.r.t. literal $\overline{x_{1}}$ and clause $C_{3}=\overline{x_{1}} \vee \overline{x_{2}} \vee x_{3}$.

Figure 3: The literal clique, for the formula $\Phi=\left(x_{1} \vee x_{2} \vee x_{3}\right) \wedge\left(\overline{x_{1}} \vee \overline{x_{2}} \vee \overline{x_{3}}\right) \wedge\left(\overline{x_{1}} \vee \overline{x_{2}} \vee x_{3}\right)$

The resulting graph $G_{\Phi}$ has diameter at most five. Indeed, all vertices but the $x_{i}, \bar{x}_{i}$ with $1 \leq i \leq n$ are adjacent to the literal clique, therefore it is a 2-distance dominating clique. We will show later how to lower the diameter (Section 3.3). Note that several vertices play almost identical roles in the reduction. This redundancy is necessary in order to ensure that most pairs of vertices that are at distance two in $G_{\Phi}$ only have one common neighbour. Indeed, the latter will impose necessary conditions on an elimination ordering of $G_{\Phi}$ to be distance-preserving. 


\subsection{Proof of correctness}

We are now ready to prove that it is NP-hard to decide whether a given graph $G$ admits a distancepreserving elimination ordering. We divide the proof in two propositions, as follows.

Proposition 5. If $\Phi$ is satisfiable, then $G_{\Phi}$ admits a distance-preserving ordering.

Proof. Let us fix a boolean assignment of the variables $x_{i}$ satisfying $\Phi$, that exists by the hypothesis. In particular, let $\left\{l_{i}, \bar{l}_{i}\right\}=\left\{x_{i}, \bar{x}_{i}\right\}$ be such that $l_{i}$ is true, let $V_{0}=\left\{\bar{l}_{i} \mid 1 \leq i \leq n\right\}$ and let $V_{1}=\left\{l_{i} \mid 1 \leq i \leq n\right\}$. Now, consider the following partition of the vertex-set of $G_{\Phi}$ into eleven subsets $S_{k}$, with $1 \leq k \leq 11$. Let $G_{0}:=G_{\Phi}$, and let $G_{k}:=G_{k-1} \backslash S_{k}$ for every $1 \leq k<11$. We will exhibit from the partition a distance-preserving ordering of $G_{\Phi}$. Precisely, we will prove that for every $1 \leq k \leq 11$ and for any total ordering $S_{k}^{\prime}$ of $S_{k}$, the elimination ordering $S_{1}^{\prime}, S_{2}^{\prime}, \ldots, S_{11}^{\prime}$ is distance-preserving.

The partition is defined as follows:

- The variable gadgets are partitioned into five subsets $S_{1}, S_{2}, S_{7}, S_{8}, S_{9}$. Furthermore, $S_{1}=$ $V_{1}=\left\{l_{i} \mid 1 \leq i \leq n\right\}, S_{8}=V_{0}=\left\{\bar{l}_{i} \mid 1 \leq i \leq n\right\}, S_{7}=\bigcup_{1 \leq i \leq n}\left\{y_{i}, \bar{y}_{i}\right\}$. The subsets $S_{2}, S_{9}$ contain the vertices $a_{i j}, b_{i j}, c_{i j}, d_{i j}$ that are respectively adjacent to a vertex of $V_{1}, V_{0}$.

- The clause tree is partitioned into four subsets $S_{3}, S_{4}, S_{5}, S_{10}$. Furthermore, $S_{5}=\left\{r_{\Phi}\right\}, S_{4}=$ $\left\{s_{1}, t_{1}, s_{2}, t_{2}, \ldots, s_{m}, t_{m}\right\}$. The subset $S_{3}$ contains the vertices $u_{j}(p, q), v_{j}(p, q)$ such that the $j^{\text {th }}$ clause is satisfied by one of $l_{p}, l_{q} \in V_{1}$; similarly, the subset $S_{10}$ contains the vertices $u_{j}(p, q), v_{j}(p, q)$ such that the $j^{\text {th }}$ clause is neither satisfied by $l_{p}$ nor $l_{q}$.

- The literal clique is partitioned into two subsets $S_{6}=\mathcal{G} \cup \mathcal{H}$ and $S_{11}=\mathcal{E} \cup \mathcal{F}$.

In what follows, we will prove that for every $1 \leq k \leq 11$, the pair $\left\langle G_{k-1}, S_{k}\right\rangle$ satisfies the sufficient condition of Corollary 3. The latter will prove, as claimed above, that for every $1 \leq k \leq 11$ and for any total ordering $S_{k}^{\prime}$ of $S_{k}$, the elimination ordering $S_{1}^{\prime}, S_{2}^{\prime}, \ldots, S_{11}^{\prime}$ is distance-preserving.

- Let $S_{1}=V_{1}=\left\{l_{i} \mid 1 \leq i \leq n\right\}$. Let $l_{i} \in S_{1}$. Neighbours of $l_{i}$ in $G_{0}$ are $y_{i}, \bar{y}_{i}$ and every of $a_{i j}, b_{i j}, c_{i j}, d_{i j}$ such that $l_{i} \in C_{j}$. Let $\alpha, \beta \in N_{G_{0}}\left(l_{i}\right)$ be non-adjacent. There are four subcases.

- if $\{\alpha, \beta\}=\left\{y_{i}, \bar{y}_{i}\right\}$ then $\bar{l}_{i}$ is a common neighbour of $\alpha, \beta$;

- if one of $\alpha, \beta$ is equal to $y_{i}$ and the other is amongst $a_{i j}, b_{i j}, c_{i j}, d_{i j}$ for some $j$, then $e_{i}, f_{i}$ are common neighbours of $\alpha, \beta$;

- similarly, if one of $\alpha, \beta$ is equal to $\bar{y}_{i}$ and the other is amongst $a_{i j}, b_{i j}, c_{i j}, d_{i j}$ for some $j$, then $\bar{e}_{i}, \bar{f}_{i}$ are common neighbours of $\alpha, \beta$;

- if $\alpha$ is amongst $a_{i j}, b_{i j}, c_{i j}, d_{i j}$ for some $j$, and $\beta$ is amongst $a_{i j^{\prime}}, b_{i j^{\prime}}, c_{i j^{\prime}}, d_{i j^{\prime}}$ for some $j^{\prime}$, then $e_{i}, f_{i}$ and $\bar{e}_{i}, \bar{f}_{i}$ are common neighbours of $\alpha, \beta$.

Therefore, in all cases $\alpha, \beta$ have a common neighbour in $G_{1}$, and Corollary 3 applies. In other words, $G_{1}=G_{\Phi} \backslash S_{1}$ is isometric and for every total ordering $S_{1}^{\prime}$ of $S_{1}$, for any prefix $S_{1}^{\prime \prime}$ of $S_{1}^{\prime}, G_{\Phi} \backslash S_{1}^{\prime \prime}$ is isometric. 
- Let $S_{2}$ contain every $a_{i j}, b_{i j}, c_{i j}, d_{i j}$ such that clause $C_{j}$ is satisfied by $l_{i}$. Let $w \in S_{2}$. There exist $j \leq m, p<q<r \leq n$ such that neighbours of $w$ in $G_{1}$ are composed of $e_{p}, f_{p}, \bar{e}_{p}, \bar{f}_{p}$ and one of $u_{j}(p, q), u_{j}(r, p), v_{j}(p, q)$ or $v_{j}(r, p)$. Let $\alpha, \beta \in N_{G_{1}}(w)$ be non-adjacent. Note that $w$ has only one neighbour in $G_{1}$ that is not in the literal clique. Consequently, one of $\alpha, \beta$ is amongst $u_{j}(p, q), u_{j}(r, p), v_{j}(p, q), v_{j}(r, p)$. Since the latter four vertices have some neighbour in the literal clique by construction, therefore $\alpha, \beta$ have a common neighbour in $G_{2}$ and Corollary 3 applies. In other words, $G_{2}=G_{1} \backslash S_{1}$ is isometric and for every total ordering $S_{2}^{\prime}$ of $S_{2}$, for any prefix $S_{2}^{\prime \prime}$ of $S_{2}^{\prime}, G_{1} \backslash S_{2}^{\prime \prime}$ is isometric.

- Let $S_{3}$ contain $u_{j}(p, q), v_{j}(p, q)$ for every $j \leq m$ and $p, q \leq n$ such that one of $l_{p}, l_{q}$ satisfies clause $C_{j}$. Let $w \in S_{3}$. There exist $j \leq m, p, q \leq n$ such that either $w=u_{j}(p, q)$ or $w=v_{j}(p, q)$. Two cases thus need to be distinguished:

- Case $w=u_{j}(p, q)$. In particular, the neighbours of $w$ in $G_{2}$ are $s_{j}, e_{p}, g_{p}, \overline{e_{q}}, \overline{g_{q}}$ and at most one amongst $a_{p j}, b_{q j}$. Furthermore, let $\alpha, \beta \in N_{G_{2}}(w)$ be non-adjacent. If $a_{p j} \in N_{G_{2}}(w)$ then $\alpha, \beta \in N_{G_{2}}\left[e_{p}\right]$, otherwise $\alpha, \beta \in N_{G_{2}}\left[\bar{e}_{q}\right]$.

- Case $w=v_{j}(p, q)$. In particular, the neighbours of $w$ in $G_{2}$ are $t_{j}, f_{p}, h_{p}, \bar{f}_{q}, \bar{h}_{q}$ and at most one amongst $c_{p j}, d_{q j}$. Furthermore, let $\alpha, \beta \in N_{G_{2}}(w)$ be non-adjacent. If $c_{p j} \in N_{G_{2}}(w)$ then $\alpha, \beta \in N_{G_{2}}\left[f_{p}\right]$, otherwise $\alpha, \beta \in N_{G_{2}}\left[\bar{f}_{q}\right]$.

In both cases, any two non-adjacent neighbours $\alpha, \beta$ of $w$ have a common neighbour in $G_{3}$ and so, Corollary 3 applies. In other words, $G_{3}=G_{2} \backslash S_{3}$ is isometric and for every total ordering $S_{3}^{\prime}$ of $S_{3}$, for any prefix $S_{3}^{\prime \prime}$ of $S_{3}^{\prime}, G_{2} \backslash S_{3}^{\prime \prime}$ is isometric.

- Let $S_{4}=\left\{s_{1}, t_{1}, s_{2}, t_{2}, \ldots, s_{m}, t_{m}\right\}$ be the vertices representing each clause. Let $w \in S_{4}$. Clearly, there exists $j \leq m$ such that either $w=s_{j}$ or $w=t_{j}$. Furthermore, by the choice of a boolean assignment satisfying $\Phi$, there exists $l_{p} \in S_{1}$ satisfying $C_{j}$. Up to cyclic permutation of the indices for the variables, this implies by construction $u_{j}(p, q), u_{j}(r, p), v_{j}(p, q), v_{j}(r, p) \in$ $S_{3}$ for some $q, r>p$. Two cases need to be distinguished.

- Case $w=s_{j}$. In particular, the neighbours of $w$ in $G_{3}$ are $r_{\Phi}$, the twelve vertices $e_{i}, g_{i}, \bar{e}_{i}, \bar{g}_{i}$ with $i \in\{p, q, r\}$, and possibly $u_{j}(q, r)$. Recall that $r_{\Phi}$ is adjacent to all the vertices of $\mathcal{G} \cup \mathcal{H}$, furthermore $u_{j}(q, r)$ is adjacent to $e_{q}, g_{q}$ and $\bar{e}_{r}, \bar{g}_{r}$. Therefore, $N_{G_{3}}(w) \subseteq N\left[g_{q}\right] \cap N\left[\bar{g}_{r}\right]$. In this situation for any two non-adjacent neighbours $\alpha, \beta$ of $w$ in $G_{3}$ we have $\alpha, \beta \in N_{G_{3}}\left[g_{q}\right]$ (resp., $\alpha, \beta \in N_{G_{3}}\left[\bar{g}_{r}\right]$ ).

Let us point out that in the full graph $G$, the two vertices $u_{j}(p, q)$ and $u_{j}(r, p)$ are also neighbours of $w=s_{j}$ in $G$. Furthermore, by construction $w$ is the unique common neighbour of $u_{j}(p, q)$ and $u_{j}(r, p)$ in $G$. Hence, it is crucial that since $\Phi$ is satisfiable, and so, $C_{j}$ is satisfied by some literal $l_{p}$, the two vertices $u_{j}(p, q)$ and $u_{j}(r, p)$ are eliminated in $S_{3}$.

- Case $w=t_{j}$. In particular, the neighbours of $w$ in $G_{3}$ are $r_{\Phi}$, the twelve vertices $f_{i}, h_{i}, \bar{f}_{i}, \bar{h}_{i}$ with $i \in\{p, q, r\}$, and possibly $v_{j}(q, r)$. Recall that $r_{\Phi}$ is adjacent to all the vertices of $\mathcal{G} \cup \mathcal{H}$, furthermore $v_{j}(q, r)$ is adjacent to $f_{q}, h_{q}$ and $\bar{f}_{r}, \bar{h}_{r}$. Therefore, $N_{G_{3}}(w) \subseteq N\left[h_{q}\right] \cap N\left[\bar{h}_{r}\right]$. In this situation for any two non-adjacent neighbours $\alpha, \beta$ of $w$ in $G_{3}$ we have $\alpha, \beta \in N_{G_{3}}\left[h_{q}\right]$ (resp., $\alpha, \beta \in N_{G_{3}}\left[\bar{h}_{r}\right]$ ).

As before, let us point out that in the full graph $G$, the two vertices $v_{j}(p, q)$ and $v_{j}(r, p)$ are also neighbours of $w=t_{j}$ in $G$. Furthermore, by construction $w$ is the unique 
common neighbour of $v_{j}(p, q)$ and $v_{j}(r, p)$ in $G$. Hence, it is crucial that since $\Phi$ is satisfiable, and so, $C_{j}$ is satisfied by some literal $l_{p}$, the two vertices $v_{j}(p, q)$ and $v_{j}(r, p)$ are eliminated in $S_{3}$.

In both cases, any two non-adjacent neighbours $\alpha, \beta$ of $w$ have a common neighbour in $G_{4}$. By Corollary $3, G_{4}=G_{3} \backslash S_{4}$ is isometric and for every total ordering $S_{4}^{\prime}$ of $S_{4}$, for any prefix $S_{4}^{\prime \prime}$ of $S_{4}^{\prime}, G_{3} \backslash S_{4}^{\prime \prime}$ is isometric.

- Let $S_{5}=\left\{r_{\Phi}\right\}$. By construction the neighbourhood of $r_{\Phi}$ in the full graph $G$ is equal to $S_{4} \cup \mathcal{G} \cup \mathcal{H}$. Note that for every $j \leq m, r_{\Phi}$ is the unique common neighbour of $s_{j}$ and $t_{j}$ in $G$, hence $G \backslash r_{\Phi}$ is not isometric. However, since all vertices in $S_{4}$ have been eliminated at this step, $r_{\Phi}$ is simplicial in $G_{4}$, i.e., its neighbourhood $N_{G_{4}}\left(r_{\Phi}\right)=\mathcal{G} \cup \mathcal{H}$ induces a complete subgraph. It is thus straightforward that Corollary 3 applies. In other words, $G_{5}=G_{4} \backslash r_{\Phi}$ is isometric.

- Let $S_{6}=\mathcal{G} \cup \mathcal{H}$. Let $w \in S_{6}$. There are four cases to be considered.

- If $w=g_{i}$ for some $i$, then neighbours of $g_{i}$ in $G_{5}$ are those in the literal clique, vertex $y_{i}$ and every $u_{j}(i, q) \notin S_{3}$. Therefore, $N_{G_{5}}[w] \subseteq N_{G_{5}}\left[e_{i}\right]$;

- if $w=\bar{g}_{i}$ for some $i$, then neighbours of $\bar{g}_{i}$ in $G_{5}$ are those in the literal clique, vertex $\bar{y}_{i}$ and every $u_{j}(p, i) \notin S_{3}$. Therefore, $N_{G_{5}}[w] \subseteq N_{G_{5}}\left[\bar{e}_{i}\right]$;

- if $w=h_{i}$ for some $i$, then neighbours of $h_{i}$ in $G_{5}$ are those in the literal clique, vertex $y_{i}$ and every $v_{j}(i, q) \notin S_{3}$. Therefore, $N_{G_{5}}[w] \subseteq N_{G_{5}}\left[f_{i}\right]$;

- else, $w=\bar{h}_{i}$ for some $i$, hence neighbours of $\overline{h_{i}}$ in $G_{5}$ are those in the literal clique, vertex $\bar{y}_{i}$ and every $v_{j}(p, i) \notin S_{3}$. Therefore, $N_{G_{5}}[w] \subseteq N_{G_{5}}\left[\bar{f}_{i}\right]$.

Since, $e_{i}, \bar{e}_{i}, f_{i}, \bar{f}_{i} \in V\left(G_{6}\right)$, therefore Corollary 3 applies. In other words, $G_{6}=G_{5} \backslash S_{6}$ is isometric and for every total ordering $S_{6}^{\prime}$ of $S_{6}$, for any prefix $S_{6}^{\prime \prime}$ of $S_{6}^{\prime}, G_{5} \backslash S_{6}^{\prime \prime}$ is isometric.

- Let $S_{7}$ contain $y_{i}, \bar{y}_{i}$ for every $1 \leq i \leq n$. Let $w \in S_{7}$. There is some $i$ such that neighbours of $w$ in $G_{6}$ are vertex $\bar{l}_{i}$ and either $e_{i}, f_{i}$ (if $w=y_{i}$ ) or $\bar{e}_{i}, \bar{f}_{i}$ (if $w=\bar{y}_{i}$ ). Moreover, recall that we assume the existence of some $1 \leq j \leq m$ such that $\bar{l}_{i}$ appears in clause $C_{j}$. Indeed, all variables are assumed to appear positively and negatively in the clauses of $\Phi$. In particular, by construction $a_{i j}, b_{i j}, c_{i j}, d_{i j} \notin S_{2}$ and so, $a_{i j}, b_{i j}, c_{i j}, d_{i j} \in V\left(G_{6}\right)$. The latter four vertices are adjacent to every of $\bar{l}_{i}, e_{i}, f_{i}$ and $\bar{e}_{i}, \bar{f}_{i}$ by construction of $G_{\Phi}$. As a result, for any $\alpha, \beta \in N_{G_{6}}(w)$ non-adjacent, $\alpha, \beta$ have a common neighbour in $G_{7}$ and so, Corollary 3 applies. In other words, $G_{7}=G_{6} \backslash S_{7}$ is isometric and for every total ordering $S_{7}^{\prime}$ of $S_{7}$, for any prefix $S_{7}^{\prime \prime}$ of $S_{7}^{\prime}, G_{6} \backslash S_{7}^{\prime \prime}$ is isometric.

- Let $S_{8}=V_{0}=\left\{\bar{l}_{i} \mid 1 \leq i \leq n\right\}$. Let $\bar{l}_{i} \in S_{8}$. Neighbours of $\bar{l}_{i}$ in $G_{7}$ are those $a_{i j}, b_{i j}, c_{i j}, d_{i j}$ such that $\bar{l}_{i}$ appears in $C_{j}$. Every such neighbour is adjacent to the 4-tuple $e_{i}, f_{i}, \bar{e}_{i}, \bar{f}_{i}$ of the literal clique, hence Corollary 3 applies. In other words, $G_{8}=G_{7} \backslash S_{8}$ is isometric and for every total ordering $S_{8}^{\prime}$ of $S_{8}$, for any prefix $S_{8}^{\prime \prime}$ of $S_{8}^{\prime}, G_{7} \backslash S_{8}^{\prime \prime}$ is isometric.

- Let $S_{9}$ contain every $a_{i j}, b_{i j}, c_{i j}, d_{i j}$ such that $\bar{l}_{i}$ appears in $C_{j}$. The proof for this case is similar as for $S_{2}$. Let $w \in S_{9}$. There are $j \leq m, p<q<r \leq n$ such that neighbours of $w$ in $G_{8}$ are $e_{p}, f_{p},{\overline{e_{p}}}_{\bar{f}}, \bar{f}_{p}$ and at most one of $u_{j}(p, q), u_{j}(r, p), v_{j}(p, q)$ or $v_{j}(r, p)$. Let $\alpha, \beta \in N_{G_{8}}(w)$ be non-adjacent. Necessarily, one of $\alpha, \beta$ must be one of $u_{j}(p, q), u_{j}(r, p), v_{j}(p, q), v_{j}(r, p)$ because 
any other neighbour of $w$ is in the literal clique. Furthermore, $u_{j}(p, q), u_{j}(r, p), v_{j}(p, q), v_{j}(r, p)$ are respectively adjacent to $e_{p}, e_{r}, f_{p}, f_{r}$ in the literal clique, that are part of $\mathcal{E} \cup \mathcal{F}$ and so, have not been eliminated with $S_{6}$. Therefore, $\alpha, \beta$ have a common neighbour in $G_{9}$ and so, Corollary 3 applies. In other words, $G_{9}=G_{8} \backslash S_{9}$ is isometric and for every total ordering $S_{9}^{\prime}$ of $S_{9}$, for any prefix $S_{9}^{\prime \prime}$ of $S_{9}^{\prime}, G_{8} \backslash S_{9}^{\prime \prime}$ is isometric.

- Let $S_{10}$ contain every $u_{j}(p, q), v_{j}(p, q)$ such that $\overline{l_{p}}, \overline{l_{q}}$ appear in $C_{j}$. Equivalently, those are all of $u_{j}(p, q), v_{j}(p, q)$ but the ones already in $S_{3}$. Let $w \in S_{10}$. There exist $j, p, q$ such that neighbours of $w$ in $G_{9}$ are either $e_{p}, \bar{e}_{q}$ (if $\left.w=u_{j}(p, q)\right)$ or $f_{p}, \bar{f}_{q}\left(\right.$ if $w=v_{j}(p, q)$ ). As a result, vertex $w$ is simplicial. It thus follows that Corollary 3 trivially applies. In other words, $G_{10}=G_{9} \backslash S_{10}$ is isometric and for every total ordering $S_{10}^{\prime}$ of $S_{10}$, for any prefix $S_{10}^{\prime \prime}$ of $S_{10}^{\prime}$, $G_{9} \backslash S_{10}^{\prime \prime}$ is isometric.

- Finally, let $S_{11}=\mathcal{E} \cup \mathcal{F}$, this is a clique and so, the vertices in $S_{11}$ can be eliminated sequentially while leaving a sequence of isometric subgraphs.

To sum up, one obtains a distance-preserving ordering of $G_{\Phi}$ by sequentially eliminating vertices in $S_{1}$ then in $S_{2}$ and so on until $S_{11}$, in an arbitrary way.

Proposition 6. If $G_{\Phi}$ admits a distance-preserving elimination ordering, then $\Phi$ is satisfiable.

Proof. Let $\prec$ be a distance-preserving ordering of $G_{\Phi}$. For every $1 \leq j \leq m$ we claim that there is $1 \leq i \leq n$ such that some $l_{i} \in\left\{x_{i}, \bar{x}_{i}\right\}$ satisfies clause $C_{j}$, and $l_{i} \prec r_{\Phi}$. Then, we will prove that this implies a boolean assignment of the variables satisfying $\Phi$ by showing that $r_{\Phi} \prec \bar{l}_{i}$, where $\left\{l_{i}, \bar{l}_{i}\right\}=\left\{x_{i}, \bar{x}_{i}\right\}$.

To prove the claim, first observe that for every $1 \leq j \leq m, r_{\Phi}$ is the unique common neighbour of $s_{j}, t_{j}$ in $G_{\Phi}$. By Corollary 2, it implies $s_{j} \prec r_{\Phi}$ or $t_{j} \prec r_{\Phi}$. So, assume $s_{j} \prec r_{\Phi}$ (the case $t_{j} \prec r_{\Phi}$ is symmetrical to this one). Let $u_{j}(p, q), u_{j}(q, r), u_{j}(r, p)$ be the three children of $s_{j}$ in the clause tree. Note that the latter three vertices pairwise share $s_{j}$ as their unique common neighbour in $G_{\Phi}$. Consequently, by Corollary 2 (applied twice) at least two of them must be eliminated before $s_{j}$. W.l.o.g., let $u_{j}(p, q)$ be eliminated before $s_{j}$. In such case, note that $u_{j}(p, q)$ is the unique common neighbour of $a_{p j}, b_{q j}$ by construction of $G_{\Phi}$. Therefore, by Corollary $2, a_{p j} \prec u_{j}(p, q)$ or $b_{q j} \prec u_{j}(p, q)$. Suppose by symmetry that $a_{p j} \prec u_{j}(p, q)$. Let $l_{p} \in\left\{x_{p}, \overline{x_{p}}\right\}$ appear in $C_{j}$. Since $l_{p}$ and $u_{j}(p, q)$ share $a_{p j}$ as their unique common neighbour and $a_{p j} \prec u_{j}(p, q)$, by Corollary 2 $l_{p} \prec a_{p j} \prec r_{\Phi}$, that finally proves the claim.

To conclude let us prove for every $1 \leq i \leq n$, there is $l_{i} \in\left\{x_{i}, \bar{x}_{i}\right\}$ such that either $l_{i} \prec r_{\Phi} \prec \overline{l_{i}}$ or $r_{\Phi} \prec l_{i} \prec \bar{l}_{i}$. If so, then let us consider any boolean assignment of the variables satisfying for every $1 \leq i \leq n, l_{i}$ is assigned true if $l_{i} \prec r_{\Phi}$ (note that if $r_{\Phi} \prec l_{i} \prec \bar{l}_{i}$, then $x_{i}$ can be valuated in an arbitrary way). Since by the above claim, for every $1 \leq j \leq m$, there is $l_{i} \prec r_{\Phi}$ satisfying clause $C_{j}$, therefore any such assignment satisfies the formula $\Phi$. By way of contradiction, suppose $l_{i} \prec \bar{l}_{i} \prec r_{\Phi}$ with $\left\{l_{i}, \overline{l_{i}}\right\}=\left\{x_{i}, \bar{x}_{i}\right\}$ for some $1 \leq i \leq n$. Since $y_{i}, \bar{y}_{i}$ share $x_{i}, \bar{x}_{i}$ as their only two common neighbours in $G_{\Phi}$, by Corollary $2 y_{i} \prec \bar{l}_{i}$ or $\bar{y}_{i} \prec \bar{l}_{i}$. Suppose by symmetry $y_{i} \prec \bar{l}_{i}$. Then, since $y_{i}$ is the unique common neighbour between $\bar{l}_{i}$ and $g_{i}, h_{i}$, we have by Corollary 2 that $g_{i} \prec y_{i}$ and $h_{i} \prec y_{i}$. However, we claim that the combination of $g_{i} \prec y_{i} \prec r_{\Phi}$ and $h_{i} \prec y_{i} \prec r_{\Phi}$ contradicts the fact that $\prec$ is distance-preserving. Indeed, $g_{i}, h_{i}$ are the only two common neighbours of $r_{\Phi}$ and $y_{i}$, so, this contradicts Corollary 2. 


\subsection{Reduction to graphs with diameter at most two}

As stated before, the graph $G_{\Phi}$ resulting from our reduction in Section 3.1 has diameter at most five. In this section, we improve the result by lowering the diameter to two, thereby proving Theorem 4 .

We base on the local view of Corollary 2, which states that in order to obtain a distancepreserving ordering of $G$ it is necessary and sufficient to ensure that vertices at distance two in $G$ still have a common neighbour in the graph at each time a vertex is eliminated. This motivates the following Definition 7 - to embed any graph $G$ into a graph $G^{\prime}$ with diameter at most two such that any two vertices at distance two in $G$ have the same set of common neighbours in $G$ and $G^{\prime}$.

Definition 7. Let $G$ be a connected graph with $n$ vertices, let $\mathcal{H}=\left\{\{u, v\} \mid u, v \in V(G)\right.$ and $\mathrm{d}_{G}(u, v) \geq$ 3 \} and let $p=|\mathcal{H}|$. The graph $G^{\prime}$ is obtained from $G$ by adding a clique $Z$ of $n+p$ vertices, defined as follows.

For every vertex $v \in V(G)$, there is $z_{v} \in Z$ that is adjacent to $v$ in $V(G)$.

For every $u, v \in V(G)$ such that $\mathrm{d}_{G}(u, v) \geq 3$, i.e., $\{u, v\} \in \mathcal{H}$, there is $z_{u v} \in Z$ that is adjacent to $u, v$ in $V(G)$.

Lemma 8. For any connected graph $G$, let $G^{\prime}$ be as in Definition $\%, G^{\prime}$ has diameter at most two.

Proof. Let $u, v \in V\left(G^{\prime}\right)$. If $u \in Z$ or $v \in Z$ then $\mathrm{d}_{G^{\prime}}(u, v) \leq 2$ because either $u, v \in Z$ are adjacent or, w.l.o.g., $u \in Z$ and $z_{v} \in Z$ is a common neighbour of $u, v$ in $G^{\prime}$ by Definition 7. Else, $u, v \in V(G)$ and so, $\mathrm{d}_{G^{\prime}}(u, v) \leq \mathrm{d}_{G}(u, v)$ because $G$ is an induced subgraph of $G^{\prime}$. Moreover, if $\mathrm{d}_{G}(u, v) \geq 3$ then by Definition 7 there is $z_{u v} \in Z$ adjacent to $u, v$ in $G^{\prime}$, therefore $\mathrm{d}_{G^{\prime}}(u, v)=2$.

Lemma 9. For any connected graph $G$, let $G^{\prime}$ be as in Definition 7, $G$ admits a distance-preserving ordering if and only if $G^{\prime}$ admits one.

Proof. Let $\left(v_{1}, v_{2}, \ldots, v_{n}\right)$ be a distance-preserving ordering of $G$. For every $1 \leq i<n$, let $G_{i}:=$ $G \backslash\left(v_{1}, \ldots, v_{i}\right)$ be an isometric subgraph of $G$, let $G_{i}^{\prime}$ be the subgraph of $G^{\prime}$ induced by $V\left(G_{i}\right) \cup Z$ (by convention, $\left.G_{0}:=G, G_{0}^{\prime}:=G^{\prime}\right)$. We claim that for every $1 \leq i<n, G_{i}^{\prime}$ is an isometric subgraph of $G^{\prime}$. Note that if the claim holds, then $\left(v_{1}, v_{2}, \ldots, v_{n}\right)$ can be completed into a distance-preserving ordering of $G^{\prime}$ as follows: vertices $v_{1}, v_{2}, \ldots, v_{n}$ are sequentially eliminated, then vertices of the clique $Z$ are eliminated in an arbitrary way ${ }^{2}$. To prove the claim, by Lemma 1 it suffices to prove that any two $x, y \in N_{G_{i-1}^{\prime}}\left(v_{i}\right)$ non-adjacent share a common neighbour in $G_{i}^{\prime}$. If $x, y \in V\left(G_{i-1}\right)$, then by Lemma 1 they share a common neighbour in $G_{i}$, hence in $G_{i}^{\prime}$. Else, one of $x, y$ is in $Z$, w.l.o.g. say $x \in Z$ and so, $z_{y} \in Z$ is a common neighbour of $x, y$ in $G_{i}^{\prime}$.

Conversely, let $G^{\prime}$ admit a distance-preserving ordering. Let $\prec$ be a distance-preserving elimination ordering of $G^{\prime}$, and let us consider the restriction $\left(v_{1}, v_{2}, \ldots, v_{n}\right)$ of the total ordering $\prec$ to the vertices of $G$. We claim that it is a distance-preserving elimination ordering of $G$. By contradiction, let $i$ be the least index such that $G_{i}:=G \backslash\left(v_{1}, v_{2}, \ldots, v_{i}\right)$ is not an isometric subgraph of $G$ (by convention, $G_{0}:=G$ ). Let $j$ be such that $v_{i}$ is the $j^{\text {th }}$ vertex to be eliminated in $G^{\prime}$ w.r.t. $\prec$, and let $G_{j}^{\prime}$ be obtained from $G^{\prime}$ by removing the $j$ first vertices to be eliminated in $G^{\prime}$ w.r.t. $\prec$. Note that $G_{j}^{\prime}$ is an isometric subgraph of $G^{\prime}$ because $\prec$ is distance-preserving by the hypothesis. Moreover, since $\left(v_{1}, v_{2}, \ldots, v_{n}\right)$ is assumed not to be distance-preserving, then by Lemma 1 , there exist $x, y \in N_{G_{i-1}}\left(v_{i}\right)$ non-adjacent whose unique common neighbour in the subgraph $G_{i-1}$ is $v_{i}$. In

\footnotetext{
${ }^{2}$ In fact, if vertices $z_{v_{1}}, z_{v_{2}}, \ldots, z_{v_{n}}$ are the last removed in $Z$ then one obtains a breadth-first search ordering rooted at $z_{v_{n}}$. This proves that the problem of deciding whether there exists a breadth-first search ordering that is distance-preserving is NP-complete.
} 
such case, $\mathrm{d}_{G}(x, y)=2$, therefore $x, y$ have no common neighbour in the clique $Z$ by Definition 7 . However, $V\left(G_{i}\right) \subseteq V\left(G_{j}^{\prime}\right) \subseteq V\left(G_{i}\right) \cup Z$ by construction, therefore $x, y$ have no common neighbour in $G_{j}^{\prime}$, that contradicts the fact that $G_{j}^{\prime}$ is an isometric subgraph of $G^{\prime}$ by Lemma 1.

Altogether, we can now prove our main result as follows.

Proof of Theorem 4. The problem is in NP. In order to prove the NP-hardness, let $\Phi$ be any instance for 3-SAT. The graph $G_{\Phi}$, described in Section 3.1, can be constructed from $\Phi$ in polynomial time. Furthermore, by the combination of Propositions 5 and $6, G_{\Phi}$ admits a distance-preserving ordering if and only if $\Phi$ is satisfiable. Finally, let $G_{\Phi}^{\prime}$ be obtained from $G_{\Phi}$ as defined in Definition 7. By Lemma $8, G_{\Phi}^{\prime}$ has diameter at most two, furthermore by Lemma $9, G_{\Phi}^{\prime}$ admits a distance-preserving ordering if and only if $G_{\Phi}$ admits one, that is if and only if $\Phi$ is satisfiable. Since 3-SAT is NPcomplete [13], this proves the hardness and so, the result.

\section{A polynomial case}

In this section, we prove that the problem of computing a distance-preserving ordering when there exists one is fixed-parameter-tractable in the treewidth.

A tree-decomposition $(T, \mathcal{X})$ of a graph $G=(V, E)$ is a pair consisting of a tree $T$ and of a family $\mathcal{X}=\left(X_{t}\right)_{t \in V(T)}$ of subsets of $V$ indexed by the nodes of $T$ and satisfying:

(i) $\bigcup_{t \in V(T)} X_{t}=V$;

(ii) for any edge $e=\{u, v\} \in E$, there exists $t \in V(T)$ such that $u, v \in X_{t}$;

(iii) for any $v \in V,\left\{t \in V(T) \mid v \in X_{t}\right\}$ induces a subtree, denoted by $T_{v}$, of $T$.

The sets $X_{t}$ are called the bags of the decomposition. Furthermore, the width of $(T, \mathcal{X})$ is equal to $\max _{t \in V(T)}\left|X_{t}\right|-1$, and the treewidth of $G$ is the minimum possible width of its tree-decompositions.

It is well-known that many NP-hard problems are fixed-parameter tractable (FPT) in the treewidth [18]. Furthermore, the existence of distance-preserving orderings has been proved useful in the comparative study of treewidth with some other properties of the tree-decompositions of graphs [17]. We prove that it can be decided in polynomial-time whether a given bounded treewidth graph admits a distance-preserving ordering, and if so, one such ordering can also be computed in polynomial-time. More precisely, we prove in what follows that the problem is FPT with the treewidth as parameter.

Theorem 10. For every $G=(V, E)$ with treewidth at most $k$, it can be decided whether a distancepreserving ordering exists in time $2^{2^{\mathcal{O}(k)}} \cdot n^{\mathcal{O}(1)}$. Furthermore, if it is the case, then a distancepreserving ordering for $G$ can also be computed within the same amount of time.

Proof. For simplicity, we will work on a specific kind of tree-decompositions, called nice treedecompositions. A tree-decomposition $(T, \mathcal{X})$ is nice if $T$ is rooted in some node $r \in V(T)$, any node of $T$ has a at most two children and, for any $t \in V(T)$,

- either $t$ is a leaf of $T$ and $\left|X_{t}\right|=1$ (Leaf Node); 
- or $t$ has one child $u$ and there exists $v \in V$ such that $X_{t}=X_{u} \backslash\{v\}$ (Forget Node);

- or $t$ has one child $u$ and there exists $v \in V$ such that $X_{t}=X_{u} \cup\{v\}$ (Introduced Node);

- or $t$ has two children $u$ and $w$ and $X_{u}=X_{w}=X_{t}$ (Join Node).

In what follows, let $(T, \mathcal{X})$ be a nice tree-decomposition of width $\mathcal{O}(k)$. It can be computed in time $2^{\mathcal{O}(k)} n$ [4]. For every $t \in V(T)$, let $T_{t}$ be the subtree rooted at node $t$ and let $V_{t}=\bigcup_{u \in T_{t}} X_{u}$. We aim at computing all the orderings on $V_{t}$ that can be extended to a distance-preserving ordering of $G$. In order to do so, we will represent an ordering on $V_{t}$ as follows:

- its subordering $\prec_{t}$ on $X_{t}$;

- the collection $\mathcal{C}_{t}$ of pairs $\left(N(v) \cap X_{t}, \operatorname{pos}_{v}\right)$ for every $v \in V_{t} \backslash X_{t}$, where $\operatorname{pos}_{v}$ is the number of neighbours in $N(v) \cap X_{t}$ preceding vertex $v$;

- finally, a set $\mathcal{P}_{t}$ of pairs $x, y \in X_{t}$ at distance two in $G$ such that both $x$ and $y$ are preceded by all their common neighbours in $V_{t}$.

Note that for any fixed vertex $v \in V_{t}$, there are $2^{\mathcal{O}(k)}$ possibilities for $N(v) \cap X_{t}$ and $\mathcal{O}(k)$ possibilities for $\operatorname{pos}_{v}$. In particular, since $\mathcal{C}_{t}$ can be any subset of a set with $\mathcal{O}(k) 2^{\mathcal{O}(k)}$ elements, there are $2^{\mathcal{O}(k) 2^{\mathcal{O}(k)}}$ possibilities for $\mathcal{C}_{t}$. Overall there are $k ! \cdot 2^{\mathcal{O}(k) 2^{\mathcal{O}(k)}} \cdot \mathcal{O}\left(k^{2}\right)=2^{2^{\mathcal{O}(k)}}$ possible representations.

Intuitively, we aim at computing for every node $t \in V(T)$ the suborderings $\prec_{t}$ of $V_{t}$ that could be potentially extended to a distance-preserving elimination ordering of $G$. In order to do so, let $\prec$ be any distance-preserving elimination ordering of $G$, let $t \in V(T)$ and let $\prec_{t}$ be the subordering of $\prec$ constrained to $V_{t}$. By Corollary 2, for every $x, y \in V_{t}$ at distance two in $G$, there exists a common neighbour $z \in N_{G}(x) \cap N_{G}(y)$ such that either $x \prec z$ or $y \prec z$. Furthermore, if $z \in V_{t}$ then we have either $x \prec_{t} z$ or $y \prec_{t} z$, otherwise since $z \notin V_{t}$ we have by the properties of a tree-decomposition that $x, y \in X_{t}$. Hence, we will consider a representation to be valid at node $t \in V(T)$ if it represents an ordering $\prec_{t}^{\prime}$ of $V_{t}$ with the following property: for every $x, y \in V_{t}$ at distance two in $G$, there exists a common neighbour $z \in N_{G}(x) \cap N_{G}(y)$ such that either $\{x, y\} \in \mathcal{P}_{t}$ and $z \in V \backslash V_{t}$, or $z \in V_{t}$ and one of $x$ or $y$ precedes $z$ w.r.t. $\prec_{t}^{\prime}$.

For every $t \in V(T)$, the following algorithm will compute all the valid representations at node $t$. Let us observe that for every subordering $\prec_{t}$ of $V_{t}$ and for any child $u \in V(T)$ of $t$, if $\prec_{t}$ has a valid representation at node $t$ then its restriction $\prec_{u}$ to $V_{u}$ also has a valid representation at node $u$. We will use this observation in what follows in order to compute the valid representations at every node by dynamic programming. Furthermore, if $\prec$ is a distance-preserving ordering of $G$ then as proved above, for every $t \in V(T)$ its restriction $\prec_{t}$ to $V_{t}$ has a valid representation at node $t$. Conversely, by Corollary 2 a valid representation at the root is equivalent to the existence of a distance-preserving ordering of $G$. Therefore, the valid representations at the root are exactly the representations of distance-preserving orderings of $G$, and so, the following algorithm is correct.

- Case of a Leaf Node. In this situation, $V_{t}=X_{t}=\{v\}$ for some $v \in V$. So, there is a unique valid representation $\left(\prec_{t}=(v), \mathcal{C}_{t}=\emptyset, \mathcal{P}_{t}=\emptyset\right)$.

- Case of a Forget Node. Let $u \in V(T)$ be the unique child of node $t$ and let $v \in V$ be such that $X_{t}=X_{u} \backslash\{v\}$. Consider any valid representation at node $u$. 
If there is a pair $\{x, v\}$ containing $v$ in $\mathcal{P}_{u}$ then we claim that it cannot be extended to a valid representation at node $t$. Indeed, since $v \in X_{u} \backslash X_{t}$ it has no neighbour in $V \backslash V_{t}$ (by Property (ii) of tree-decompositions). Therefore, given any subordering on $V_{t}$ that is mapped to this representation, the two vertices $v$ and $x$ are eliminated after all their common neighbours in any extension of this subordering to a total ordering on $V$. The latter falsifies the characterization of distance-preserving orderings given in Corollary 2, that proves the claim.

Else, there is no pair of $\mathcal{P}_{u}$ containing $v$. In this situation, the representation can be transformed into a valid representation at node $t$ by taking the restriction of $\prec_{u}$ to $X_{t}$ and by constructing $\mathcal{C}_{t}$ as follows. First let us add the pair $\left(N(v) \cap X_{t}, \operatorname{pos}_{v}\right)$ in $\mathcal{C}_{t}$, that can be easily computed from $\prec_{u}$ (recall that $\operatorname{pos}_{v}$ is the number of neighbours of $v$ in $X_{u}$ that are preceding $v$ w.r.t. $\left.\prec_{u}\right)$. Then for every pair $(N, p) \in \mathcal{C}_{u}$, either $v$ is among the $p$ first neighbours in $N$ w.r.t. $\prec_{u}$, in which case let us add $(N \backslash v, p-1)$ in $\mathcal{C}_{t}$, or let us add $(N \backslash v, p)$ in $\mathcal{C}_{t}$.

- Case of an Introduced Node. Let $u \in V(T)$ be the unique child of node $t$ and let $v \in V$ be such that $X_{t}=X_{u} \cup\{v\}$. Consider any valid representation at node $u$. We consider the $\mathcal{O}(k)$ possible ways to insert $v$ w.r.t. $\prec_{u}$, in order to obtain the subordering $\prec_{t}$. For every $\prec_{t}$, we need to consider all vertices in $V_{t}$ that are at distance two from $v$. We distinguish between two subcases.

- First, let $Y_{u} \subseteq X_{u}$ contain all the vertices $x$ of $X_{u}$ that are at distance two from $v$. For every $x \in Y_{u}$, we check whether there exists a common neighbour $z$ such that either $z \in X_{u}$ and it is preceded by one of $x$ or $v$ (this can be checked with $\prec_{t}$ ), or $z \notin V_{t}$.

If no such vertex $z$ exists (that means that all common neighbours are in $V_{t}$ and they all preceed $x$ and $v$ in the current ordering) then we claim that it is not possible to extend to a valid representation at node $t$. Indeed, let us fix an arbitrary extension $\prec$ of $\prec_{t}$ to a total ordering on $V$. Suppose by way of contradiction that $\prec$ is distance-preserving. By Corollary 2, there exists $z^{\prime} \in N(v) \cap N(x)$ such that $x \prec z^{\prime}$ or $v \prec z^{\prime}$. Furthermore, $z^{\prime} \in V_{t}$ (else, we could choose $z=z^{\prime}$, that is a contradiction). By Property (ii) of tree-decompositions, $v$ has no neighbours in $V_{t} \backslash X_{u}$, and so, $z^{\prime} \in X_{u}$. However, since $\prec$ is an extension of $\prec_{t}$, the latter implies that $x \prec_{t} z^{\prime}$ or $v \prec_{t} z^{\prime}$. Hence we could choose $z=z^{\prime}$, that is a contradiction. Therefore, the claim is proved.

Otherwise, there exists a common neighbour $z$ as defined above. In this situation, we will need to add the pair $\{x, v\}$ in $\mathcal{P}_{t}$ if and only if all possible choices for $z$ are in $V \backslash V_{t}$. Note that after iterating on all the vertices of $Y_{u}$, we will also need to complete $\mathcal{P}_{t}$ with the pairs $\{x, y\} \in \mathcal{P}_{u}$ such that $x$ and $y$ have a common neighbour in $V \backslash V_{t}$ and they are preceded by all their common neighbours in $V_{t}=V_{u} \cup\{v\}$. Furthermore, we need to check that for all the pairs $\{x, y\} \in \mathcal{P}_{u} \backslash \mathcal{P}_{t}$, vertex $v$ is a common neighbour of $x$ and $y$ such that either $x \prec_{t} v$ or $y \prec_{t} v$ (otherwise, we cannot extend to a valid representation and $\prec_{t}$ can be discarded).

- Second, let us consider all vertices $x \in V_{t} \backslash X_{u}$ that are at distance two from $v$. Note that since by Property (ii) of tree-decompositions $v$ has no neighbours in $V_{t} \backslash X_{u}$, we have that for every $x \in V_{t} \backslash X_{u}, x$ is at distance two from $v$ if and only if $\left(N(x) \cap X_{u}\right) \cap N(v) \neq \emptyset$. Precisely, all the common neighbours of $v$ and $x$ are in $X_{u}$ (and in $X_{t}$ ). So, let us 
consider all the pairs $(N, p) \in \mathcal{C}_{u}$ such that $N \cap N(v) \neq \emptyset$ (intuitively, this corresponds to a vertex $x \in V_{t} \backslash X_{u}$ that has a common neighbour with $v$ ).

For every such pair $(N, p)$, let us define $N^{+}$as the subset obtained from $N$ by removing its $p$ first vertices w.r.t. $\prec_{u}$. Similarly, let $N^{+}(v)$ be the vertices of $N(v) \cap X_{u}$ that are preceded by $v$ w.r.t. $\prec_{t}$. We check whether either $N^{+}(v) \cap N \neq \emptyset$ or $N^{+} \cap N(v) \neq \emptyset$. Intuitively, the former corresponds to the case where $v$ preceeds one common neighbour of $x$ and $v$, and the latter corresponds to the case where $x$ preceeds one common neighbour of $x$ and $v$, with $x$ being such that $\left(N(x) \cap X_{u}, p o s_{x}\right)=(N, p)$. If the test fails then we claim that we cannot extend to a valid representation at node $t$ (and so, the current subordering $\prec_{t}$ can be discarded).

Indeed, let $\prec$ be any extension of $\prec_{t}$ to a total ordering on $V$. Suppose by way of contradiction that $\prec$ is distance-preserving. Let $x \in V_{t} \backslash X_{u}$ be such that $(N(x) \cap$ $\left.X_{u}, \operatorname{pos}_{x}\right)=(N, p)$. Note that $x$ and $v$ are at distance two. So, by Corollary 2, there exists a common neighbour $z \in X_{u}$ that is preceded by at least one of $v$ or $x$. Furthermore, let us denote by $N^{+}(x)=N^{+}$the subset of $N(x) \cap X_{u}$ obtained by removing its $\operatorname{pos}_{x}$ first neighbours in $X_{u}$ w.r.t. $\prec_{t}$. We get that either $v$ precedes $z$, and so, $N^{+}(v) \cap N(x) \neq$ $\emptyset$, or $x$ precedes $z$, and so, $N^{+}(x) \cap N(v) \neq \emptyset$. The latter contradicts that neither $N^{+}(v) \cap N \neq \emptyset$ nor $N^{+} \cap N(v) \neq \emptyset$, therefore the claim is proved.

Conversely, let us point out that if for every $(N, p) \in \mathcal{C}_{u}$ such that $N \cap N(v) \neq \emptyset$, either $N^{+}(v) \cap N \neq \emptyset$ or $N^{+} \cap N(v) \neq \emptyset$, then the following holds for every $x \in V_{t} \backslash X_{u}$ at distance two from $v$ : either $N^{+}(v) \cap N(x) \neq \emptyset$, and so, there exists a common neighbour $z$ preceded by $v$, or $N^{+}(x) \cap N(v) \neq \emptyset$, and so, there exists a common neighbour $z$ preceded by $x$.

Note that the collection $\mathcal{C}_{u}=\mathcal{C}_{t}$ is not modified.

- Case of a Join Node. Let $u, w$ be the two children nodes of $t$. Recall that $X_{u}=X_{w}=X_{t}$. Consider any valid representation at node $u$, and any valid representation at node $w$. They can be merged into a valid representation at node $t$ only if $\prec_{u}=\prec_{w}$. If so, let $\prec_{t}=\prec_{u}$, let $\mathcal{P}_{t}=\mathcal{P}_{u} \cap \mathcal{P}_{w}$ and let $\mathcal{C}_{t}=\mathcal{C}_{u} \cup \mathcal{C}_{w}$.

In order to decide whether this can be extended into a valid representation at node $t$, we need to consider all the pairs of vertices in $V_{t}$ at distance two in $G$ that are neither both contained in $V_{u}$ nor both contained in $V_{w}$. More precisely, we need to consider all the pairs of vertices $v_{u} \in V_{u} \backslash X_{u}, v_{w} \in V_{w} \backslash X_{w}$ at distance two in $G$. Notice that since by Property (ii) of tree-decompositions, there cannot be an edge between $V_{u} \backslash X_{u}$ and $V_{w} \backslash X_{w}$, the pairs $v_{u} \in V_{u} \backslash X_{u}, v_{w} \in V_{w} \backslash X_{w}$ that need to be considered are exactly those such that $\left(N\left(v_{u}\right) \cap\right.$ $\left.X_{u}\right) \cap\left(N\left(v_{w}\right) \cap X_{w}\right) \neq \emptyset$. Hence, let us consider all the pairs $\left(N_{u}, p_{u}\right) \in \mathcal{C}_{u},\left(N_{w}, p_{w}\right) \in \mathcal{C}_{w}$ such that $N_{u} \cap N_{w} \neq \emptyset$.

For every two pairs $\left(N_{u}, p_{u}\right) \in \mathcal{C}_{u},\left(N_{w}, p_{w}\right) \in \mathcal{C}_{w}$ such that $N_{u} \cap N_{w} \neq \emptyset$, let $N_{u}^{+}$be the subset obtained from $N_{u}$ by removing its $p_{u}$ first vertices w.r.t. $\prec_{t}=\prec_{u}$. Intuitively, $N_{u}^{+}$ corresponds to the neighbours of some vertex $v_{u} \in V_{u} \backslash X_{u}$ that are in $X_{u}=X_{t}$ and preceded by $v_{u}$. Similarly, let $N_{w}^{+}$be the subset obtained from $N_{w}$ by removing its $p_{w}$ first vertices w.r.t. $\prec_{t}=\prec_{w}$. We check whether either $N_{u}^{+} \cap N_{w} \neq \emptyset$ or $N_{w}^{+} \cap N_{u} \neq \emptyset$. If it is not the case then we claim that we cannot extend to a valid representation at node $t$ (and so, the current subordering $\prec_{t}$ can be discarded). 
Indeed, let $\prec$ be any extension of $\prec_{t}$ to a total ordering on $V$. Suppose by way of contradiction that $\prec$ is distance-preserving. Let $v_{u} \in V_{u} \backslash X_{u}, v_{w} \in V_{w} \backslash X_{w}$ be such that $\left(N\left(v_{u}\right) \cap\right.$ $\left.X_{u}, \operatorname{pos}_{v_{u}}\right)=\left(N_{u}, p_{u}\right)$ and $\left(N\left(v_{w}\right) \cap X_{w}, \operatorname{pos}_{v_{w}}\right)=\left(N_{w}, p_{w}\right)$. Since $N\left(v_{u}\right) \cap N\left(v_{w}\right)=N_{u} \cap$ $N_{w} \neq \emptyset, v_{u}$ and $v_{w}$ are at distance two. Therefore, by Corollary 2, there exists a common neighbour $z \in X_{t}$ that is preceded by at least one of $v_{u}$ or $v_{w}$. Furthermore, let us denote by $N^{+}\left(v_{u}\right)=N_{u}^{+}$the subset of $N\left(v_{u}\right) \cap X_{u}$ obtained by removing its $\operatorname{pos}_{v_{u}}$ first neighbours in $X_{u}$ w.r.t. $\prec_{t}=\prec_{u}$; similarly, let us denote by $N^{+}\left(v_{w}\right)=N_{w}^{+}$the subset of $N\left(v_{w}\right) \cap X_{w}$ obtained by removing its $\operatorname{pos}_{v_{w}}$ first neighbours in $X_{w}$ w.r.t. $\prec_{t}=\prec_{w}$. We get that either $v_{u}$ precedes $z$, and so, $N^{+}\left(v_{u}\right) \cap N\left(v_{w}\right) \neq \emptyset$, or $v_{w}$ precedes $z$, and so, $N^{+}\left(v_{w}\right) \cap N\left(v_{u}\right) \neq \emptyset$. The latter contradicts that neither $N_{u}^{+} \cap N_{w} \neq \emptyset$ nor $N_{w}^{+} \cap N_{u} \neq \emptyset$, therefore the claim is proved.

Conversely, let us point out that if for every $\left(N_{u}, p_{u}\right) \in \mathcal{C}_{u},\left(N_{w}, p_{w}\right) \in \mathcal{C}_{w}$ such that $N_{u} \cap N_{w} \neq$ $\emptyset$, either $N_{u}^{+} \cap N_{w} \neq \emptyset$ or $N_{w}^{+} \cap N_{u} \neq \emptyset$, then the following holds for every $v_{u} \in V_{u} \backslash X_{u}, v_{w} \in$ $V_{w} \backslash X_{w}$ at distance two in $G$ : either $N^{+}\left(v_{u}\right) \cap N\left(v_{w}\right) \neq \emptyset$, and so, there exists a common neighbour $z$ preceded by $v_{u}$, or $N^{+}\left(v_{w}\right) \cap N\left(v_{u}\right) \neq \emptyset$, and so, there exists a common neighbour $z$ preceded by $v_{w}$.

\section{$5 \quad$ Exact algorithms and heuristics}

The purpose of the section is to describe algorithms in order to compute a distance-preserving ordering for a given graph $G$ when it exists. Exhaustive-search on all possible vertex-orderings of the graph would require $\mathcal{O}^{*}(n !)=2^{\mathcal{O}(n \log n)}$-time ${ }^{3}$, and the algorithm parameterized by treewidth that we have presented in Section 4 has huge constants which makes it rather impractical.

In this section, we describe exact and heuristic algorithms that can effectively be used to decide if a graph has a distance-preserving ordering, and return one when it exists.

\subsection{Exact exponential time algorithm}

A meta-theorem for computing vertex-orderings in graphs with given properties was proved in [3]. It bases on dynamic programming. Here, we prove that the theorem of [3] also applies to distancepreserving orderings. For any elimination ordering $\left(v_{1}, v_{2}, \ldots, v_{n}\right)$ of a graph $G=(V, E)$ and for any $1 \leq i \leq n$, let $V_{i+1}=\left\{v_{i+1}, v_{i+2}, \ldots, v_{n}\right\}=\left\{u \in V \mid v_{i} \prec u\right\}$.

Theorem 11 ( [3]). Let $f$ be a polynomial time computable function mapping each 3-tuple, consisting of a graph $G=(V, E)$, a vertex set $S \subseteq V$, and a vertex $v \in V$ to an integer.

Then we can compute in $\mathcal{O}^{*}\left(2^{n}\right)$-time and space, or in $\mathcal{O}^{*}\left(4^{n}\right)$-time and polynomial-space, the following values for a given graph $G=(V, E)$ :

- $\min _{\prec} \max _{v_{i} \in V} f\left(G, V_{i+1}, v_{i}\right)$;

- $\min _{\prec} \sum_{v_{i} \in V} f\left(G, V_{i+1}, v_{i}\right)$.

\footnotetext{
${ }^{3}$ The notation $\mathcal{O}^{*}(f(n))$ is for a complexity $f(n) \cdot n^{\mathcal{O}(1)}$
} 
Corollary 12. The problem of deciding whether a given graph admits a distance-preserving elimination ordering can be solved in $\mathcal{O}^{*}\left(2^{n}\right)$-time and space, or in $\mathcal{O}^{*}\left(4^{n}\right)$-time and polynomial-space.

Proof. Let the function $f$ map every 3-tuple $(G, S, v)$ to the number of pairs $x, y \in S \cap N_{G}(v)$ of nonadjacent vertices with no common neighbour in $S$. Given a graph $G=(V, E)$ our aim is to compute an elimination ordering $\left(v_{1}, v_{2}, \ldots, v_{n}\right)$ of $G$ that minimizes $\max _{1<i<n} f\left(G, V_{i+1}, v_{i}\right)$, with $V_{i+1}=\left\{v_{i+1}, v_{i+2}, \ldots, v_{n}\right\}$. Indeed, by Corollary 2, $G$ admits a distance-preserving elimination ordering if and only if there is one such ordering such that for every $1 \leq i \leq n, f\left(G, V_{i+1}, v_{i}\right)=0$, i.e., $\max _{1 \leq i<n} f\left(G, V_{i+1}, v_{i}\right)=0$. By Theorem 11 and since $f$ is polynomial-time computable an ordering that minimizes $\max _{1 \leq i<n} f\left(G, V_{i+1}, v_{i}\right)$ can be computed in $\mathcal{O}^{*}\left(2^{n}\right)$-time and space, or in $\mathcal{O}^{*}\left(4^{n}\right)$-time and polynomial-space.

\section{$5.2 \quad$ Integer linear programming}

Integer linear programming (ILP) formulations have been proved useful in practical computation of vertex orderings $[8,16]$. For completeness, we hence propose an ILP formulation that fits to our problem. Like in [16], total ordering on the vertices is expressed through $n^{2}$ binary variables $x_{v, i}$, each denoting whether vertex $v \in V$ is amongst the $i$ first vertices to be eliminated.

$$
\begin{array}{rlrl}
\sum_{v \in V} x_{v, i} & =i & & \forall 1 \leq i \leq n \\
x_{v, i} \leq x_{v, i+1} & \forall v \in V, \forall 1 \leq i<n
\end{array}
$$

In order to ensure that the total ordering is distance-preserving, we impose that for all pairs of vertices $u, v \in V$ at distance two in $G$, at least one of $u$ or $v$ must be eliminated before some of their common neighbours $w$. It can be expressed as follows:

$$
\begin{aligned}
& \sum_{w \in N_{G}(u) \cap N_{G}(v)} x_{w, i} \leq x_{u, i}+x_{v, i}+\left(\left|N_{G}(u) \cap N_{G}(v)\right|-1\right) \\
& \forall u, v \text { s.t. } \mathrm{d}_{G}(u, v)=2, \forall 1 \leq i \leq n
\end{aligned}
$$

The correctness of our formulation directly follows from Corollary 2.

\subsection{Heuristics}

In this section, we present three heuristics to decide whether a graph admits a distance-preserving ordering. Then, we propose two ways to generate graphs admitting distance-preserving orderings.

Heuristic Greedy_Pruning The first heuristic, very naive, attempts to find a distance preserving ordering greedily. Precisely, given a graph $G$, it computes the set $C$ of all vertices $v$ such that $G \backslash v$ is an isometric subgraph of $G$. Note that, by Lemma 1, this can be done by checking only the vertices at distance at most two for every vertex in $G$. Once the set $C$ of candidates has been computed, one vertex $v$ is randomly chosen in it (this will be the first vertex of the tried ordering) and the process goes on $G \backslash v$. If $C=\emptyset$, the process stops and returns that no distance preserving ordering has been found. If $G$ has no more vertices, the algorithm returns the found ordering. The pseudo-code of this heuristic is presented in Algorithm 1. 


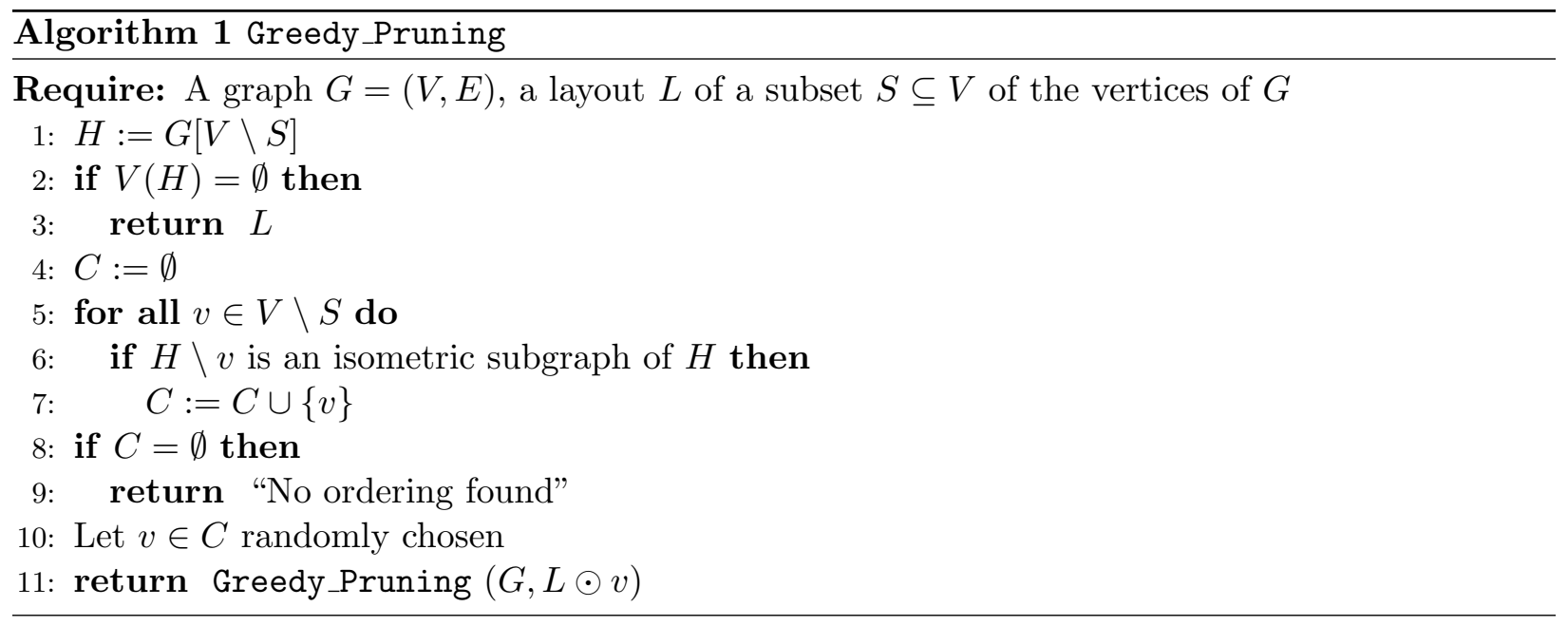

Heuristic Greedy_Reverse_Pruning The second heuristic attempts to build the ordering starting from its last vertex. Precisely, it guesses the last vertex (all vertices of the graph $G$ may be considered as last vertex). From the current vertex, the algorithm tries to guess its predecessor in the ordering. Precisely, assuming that the algorithm has already computed a partial layout $\left(v_{i+1}, \cdots, v_{n}\right)$ of a set $S=\left\{v_{i+1}, \cdots, v_{n}\right\} \subseteq V$, it aims at finding a vertex $v_{i}$ such that $G[S]$ is an isometric subgraph of $G\left[\left\{v_{i}\right\} \cup S\right]$. For this purpose, it computes the set $C$ of all vertices $v \in N(S)=\{v \in V(G) \backslash S \mid \exists u \in S,\{u, v\} \in E(G)\}$ that satisfies this property. By Lemma 1, a vertex $v \in N(S)$ is added to $C$ if any two non-adjacent neighbours $x, y \in N(v) \cap S$ have another common neighbour in $S$. Once the set $C$ of candidates has been computed, if it is empty, then the process stops and returns that no distance preserving ordering has been found. Otherwise, there are two variants of the heuristic:

- In the first one, one vertex $v$ is randomly chosen in $C$.

- In the second case, one vertex $v$ is randomly chosen in the set of the vertices of $C$ that have maximum degree in $S$.

In both cases, the chosen vertex $v$ is added as first vertex of the current layout. The pseudo-code of this heuristic (second variant) is presented in Algorithm 2. For the first variant, the only difference is that Line 9 must be replaced by "Let $C^{*}:=C$ ".

The intuition behind the fact that it seems preferable to take a vertex with maximum degree in $S$ (Line 9 of Algorithm 2) is clear since it will maximize the number of pairs of vertices already having a common neighbour in $S$ (which is a required condition to compute $C$ on Line 5 of Algorithm 2).

Graph generation To generate graphs with distance preserving ordering (in order to test the heuristics), we propose the following two algorithms.

- The first algorithm (INC) creates a graph by adding the vertices one by one. Precisely, assuming that a graph $G$ (admitting a distance-preserving ordering) has already been created, the algorithm adds a new vertex as follows. First a vertex $x \in V(G)$ is randomly chosen. Then, the algorithm randomly chooses a set $X \subseteq\{w \in V(G) \mid \operatorname{dist}(x, w) \leq 2\}$ with the property that any two non-adjacent vertices in $X$ have a common neighbour is $G$. Finally, a 


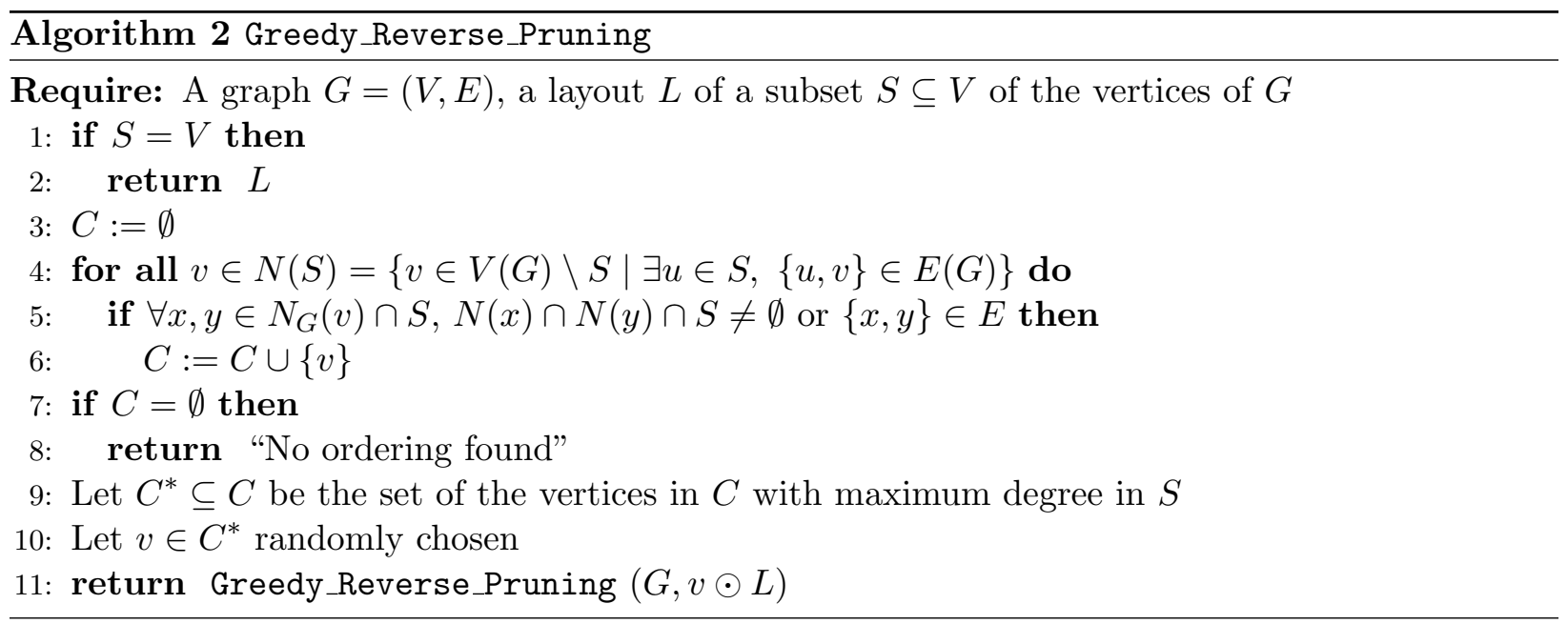

new vertex $v$ is added to $G$ by making $v$ adjacent to every vertex in $X \cup\{x\}$. The cardinality of $X$ can additionally be bounded.

- The second algorithm (AUG) aims at augmenting a given graph into a super-graph of it admitting a distance-preserving ordering. For this purpose, the algorithm starts from a given graph $G=(V, E)$ and first computes a random ordering $L=\left(v_{1}, \cdots, v_{n}\right)$ of $V$. Then, we aim at adding edges to $G$ in order to make $L$ a distance-preserving ordering of the resulting super-graph of $G$. Precisely, the algorithm considers the vertices one by one from $v_{1}$ to $v_{n}$. When considering $v_{i}$, if $G_{i+1}=G\left[\left\{v_{i+1}, \cdots, v_{n}\right\}\right]$ is an isometric subgraph of $G_{i}$, then no edges are added. Otherwise, by Lemma 1, this means that two non-adjacent neighbours $x, y \in V\left(G_{i+1}\right) \cap N\left(v_{i}\right)$ of $v_{i}$ have no common neighbour in $G_{i+1}$. Hence, the algorithm adds edges between every such a pair of vertices.

We have used these generators to perform basic experiments on a standard laptop, using the Sagemath open-source mathematical software [30] to implement the algorithms and IBM Ilog CPLEX [23] to solve the ILP formulations. Our first observation is that the ILP formulation is generally able to decide if a graph with up to 50 nodes has a distance-preserving ordering in a few minutes (we also tried Erdős-Rényi and Barabási-Albert random graphs). However, it can hardly be used for larger graphs due to excessive running time. Our second observation is that the Greedy_Pruning heuristic is not effective at all. It is able to find a distance-preserving ordering on very few small graphs (less than 20 nodes) only. The Greedy_Reverse_Pruning heuristic guided by the maximum degree is much more efficient. We have executed it on graphs generated by the INC generator (100 n-node graphs, for each $n \in\{20,30, \cdots, 100\})$. The heuristic has been able to confirm that more than $96 \%$ of these graphs have a distance-preserving ordering. Also, this heuristic appears to be particularly efficient on dense graphs. Precisely, we performed many experiments on Erdős-Rényi random graphs (100n-node graphs, for each $n \in\{100, \cdots, 200\}$ and $p \in\{0.1,0.2, \cdots, 0.5\})$ and our heuristic returns that more than $99 \%$ of them actually have a distance-preserving ordering when the probability is high $(p \geq 0.3)$. The latter supports a recent conjecture from [27]. Further experimental and theoretical investigations are needed to determine the minimum probability upon which Erdös-Rényi random graphs have a distance-preserving ordering asymptotically almost surely. We let this interesting question as an open problem for future 
research.

\section{Acknowledgments}

We wish to thank the referees for their careful reading of the first version of this manuscript, their useful comments, and for pointing out the relationship between the distance-preserving orderings of hypercubes and the shellable orderings of cross-polytopes.

\section{References}

[1] H.-J. Bandelt and V. Chepoi. Metric graph theory and geometry: a survey. Contemporary Mathematics, 453:49-86, 2008.

[2] H.-J. Bandelt and H. Mulder. Distance-hereditary graphs. Journal of Combinatorial Theory, Series B, 41(2):182-208, 1986.

[3] H. Bodlaender, F. Fomin, A. Koster, D. Kratsch, and D. Thilikos. A note on exact algorithms for vertex ordering problems on graphs. Theory of Computing Systems, 50(3):420-432, 2012.

[4] H. L. Bodlaender, P. G. Drange, M. S. Dregi, F. V. Fomin, D. Lokshtanov, and M. Pilipczuk. A $c^{k} n$ 5-approximation algorithm for treewidth. SIAM Journal on Computing, 45(2):317-378, 2016 .

[5] J. Bondy and U. Murty. Graph theory with applications, volume 290. Macmillan London, 1976.

[6] A. Brandstädt, F. Dragan, V. Chepoi, and V. Voloshin. Dually chordal graphs. SIAM Journal on Discrete Mathematics, 11(3):437-455, 1998.

[7] A. Brandstädt, F. Dragan, and F. Nicolai. Homogeneously orderable graphs. Theoretical Computer Science, 172(1):209-232, 1997.

[8] A. Cassioli, O. Günlük, C. Lavor, and L. Liberti. Discretization vertex orders in distance geometry. Discrete Applied Mathematics, 197:27-41, 2015.

[9] J. Chalopin, V. Chepoi, H. Hirai, and D. Osajda. Weakly modular graphs and nonpositive curvature. arXiv preprint arXiv:1409.3892, to appear in Memoirs of AMS, 2014.

[10] J. Chalopin, V. Chepoi, N. Nisse, and Y. Vaxes. Cop and robber games when the robber can hide and ride. SIAM Journal on Discrete Mathematics, 25(1):333-359, 2011.

[11] V. Chepoi. On distance-preserving and domination elimination orderings. SIAM Journal on Discrete Mathematics, 11(3):414-436, 1998.

[12] N. Clarke and R. Nowakowski. Tandem-win graphs. Discrete Mathematics, 299(1):56-64, 2005.

[13] S. Cook. The complexity of theorem-proving procedures. In Proceedings of the Third Annual ACM Symposium on Theory of Computing, STOC '71, pages 151-158, New York, NY, USA, 1971. ACM. 
[14] D. Corneil and M. Habib. Unified view of graph searching and LDFS-based certifying algorithms. In Encyclopedia of Algorithms. 2015.

[15] D. Corneil and J. Stacho. Vertex ordering characterizations of graphs of bounded asteroidal number. Journal of Graph Theory, 78(1):61-79, 2015.

[16] D. Coudert. A note on Integer Linear Programming formulations for linear ordering problems on graphs. Research report, Inria ; I3S ; Universite Nice Sophia Antipolis ; CNRS, Feb. 2016.

[17] D. Coudert, G. Ducoffe, and N. Nisse. To approximate treewidth, use treelength! SIAM Journal on Discrete Mathematics, 30(3):1424-1436, 2016.

[18] B. Courcelle. The monadic second-order logic of graphs. I. recognizable sets of finite graphs. Information and computation, 85(1):12-75, 1990.

[19] E. Dahlhaus, P. Hammer, F. Maffray, and S. Olariu. On domination elimination orderings and domination graphs. In Graph-Theoretic Concepts in Computer Science, pages 81-92. Springer, 1995.

[20] R. Diestel. Graph theory, volume 173 of Graduate texts in mathematics. Springer, Heidelberg, 1997.

[21] M. Habib and C. Paul. A simple linear time algorithm for cograph recognition. Discrete Applied Mathematics, 145(2):183-197, 2005.

[22] H. T. Hall. Counterexamples in discrete geometry. PhD thesis, University of California, Berkeley, 2004.

[23] IBM/ILOG. CPLEX Optimizer, version 12.7, 2016.

[24] B. Jamison and S. Olariu. On the semi-perfect elimination. Advances in applied mathematics, 9(3):364-376, 1988.

[25] R. Nowakowski and P. Winkler. Vertex-to-vertex pursuit in a graph. Discrete Mathematics, 43(2):235-239, 1983.

[26] R. Nussbaum, A. Esfahanian, and P. Tan. Clustering social networks using distance-preserving subgraphs. In The Influence of Technology on Social Network Analysis and Mining, volume 6 of Lecture Notes in Social Networks, pages 331-349. Springer, 2013.

[27] R. Nussbaum and A.-H. Esfahanian. Preliminary results on distance-preserving graphs. Congressus Numerantium, 211:141-149, 2012.

[28] D. Rose, R. Tarjan, and G. Lueker. Algorithmic aspects of vertex elimination on graphs. SIAM Journal on computing, 5(2):266-283, 1976.

[29] J. P. Smith and E. Zahedi. On distance preserving and sequentially distance preserving graphs. arXiv preprint arXiv:1701.06404, 2017.

[30] W. A. Stein et al. Sage Mathematics Software System (Version 7.5). The Sage Development Team, 2017. 
[31] M. Tancer. Recognition of collapsible complexes is NP-complete. Discrete \& Computational Geometry, 55(1):21-38, 2016.

[32] A. Vince. A non-shellable 3-sphere. European Journal of Combinatorics, 6(1):91-100, 1985. 